\title{
Multivariate analysis of Kelvin wave seasonal variability in ECMWF L91 analyses
}

\author{
Marten Blaauw and Nedjeljka Žagar \\ University of Ljubljana, Faculty of mathematics and physics, Ljubljana, Slovenia \\ Correspondence: Marten Blaauw (marten.blaauw@fmf.uni-lj.si)
}

Received: 30 January 2018 - Discussion started: 21 February 2018

Revised: 6 May 2018 - Accepted: 24 May 2018 - Published: 14 June 2018

\begin{abstract}
This paper presents a multivariate analysis of the linear Kelvin waves (KWs) represented by the operational 91-level ECMWF analyses in the 2007-2013 period, with a focus on seasonal variability. The applied method simultaneously filters $\mathrm{KW}$ wind and temperature perturbations in the continuously stratified atmosphere on the spherical Earth. The spatial filtering of the three-dimensional KW structure in the upper troposphere and lower stratosphere is based on the Hough harmonics using several tens of linearized shallowwater equation systems on the spherical Earth with equivalent depths ranging from $10 \mathrm{~km}$ to a few metres.

Results provide the global KW energy spectrum. It shows a clear seasonal cycle with the KW activity predominantly in zonal wavenumbers $1-2$, where up to $50 \%$ more energy is observed during the solstice seasons in comparison with boreal spring and autumn.

Seasonal variability in KWs in the upper troposphere and lower stratosphere is examined in relation to the background wind and stability. A spectral bandpass filtering is used to decompose variability into three period ranges: seasonal, intraseasonal and intra-monthly variability components. Results reveal a slow seasonal KW component with a robust dipole structure in the upper troposphere with its position determined by the location of the dominant convective outflow throughout the seasons. Its maximal strength occurs during boreal summer when easterlies in the eastern hemisphere are strongest. The two other components represent vertically propagating KWs and are observed throughout the year with seasonal variability mostly found in the wave amplitudes being dependent on the seasonality of the background easterly winds and static stability.
\end{abstract}

\section{Introduction}

Atmospheric equatorial Kelvin waves (hereafter KWs), first discovered in the stratosphere (Wallace and Kousky, 1968), are nowadays observed and studied over a broad range of spatial and temporal scales. A broad wavenumber-frequency spectrum can be traced to the spatiotemporal nature of tropical convection which generates KWs along with a spectrum of other equatorial waves. The atmospheric wave response to the stochastic nature of convection was studied by Garcia and Salby (1987) and Salby and Garcia (1987) who made a distinction between (i) projection or vertical response to short-term heating fluctuations (e.g. daily convection) and (ii) the barotropic or horizontal response to seasonal convective heating. For KWs, the vertical response gives rise to a broad frequency spectrum of vertically propagating KWs that radiate outward into the stratosphere where they drive zonalmean quasi-periodic flows such as the quasi-biennial oscillation (QBO; Holton and Lindzen, 1972). The horizontal response to seasonal transitions in convective heating gives rise to planetary-scale disturbances with a half-sinusoidal vertical structure confined to the troposphere. A part of this response remains stationary over the convective hotspot; its shape resembling a classic "Gill-type" KW solution (Gill, 1980). The other part of the response intensifies and advances over the Pacific, representing a transient component of the Walker circulation (Salby and Garcia, 1987).

Both components of the KW response received increased attention in the scientific community over the last decades in terms of the role they play in the (intra-)seasonal variability in the tropical tropopause layer (hereafter TTL), defined as a transition layer between the typical level of convective outflow at $\sim 12 \mathrm{~km}$, where the Brunt-Väisälä frequency is at its minimum, and the cold point tropopause 
at $\sim 16-17 \mathrm{~km}$ (Highwood and Hoskins, 1998; Fueglistaler et al., 2009). Within the TTL, temperature variations play an important role in controlling the stratosphere-troposphere exchange of various species such as ozone and water vapour, thereby aiding in the dehydration process of air entering the stratosphere. The two parts of the KW response modulate the TTL differently on different timescales (Highwood and Hoskins, 1998; Randel and Wu, 2005; Ryu et al., 2008; Flannaghan and Fueglistaler, 2013); their relative contribution to TTL dynamics varies with season and is not yet fully understood. The present study contributes to this topic by applying a novel multivariate analysis of $\mathrm{KW}$ seasonal variability in model-level analysis data.

Seasonal variations in KW dynamics in the TTL have been previously studied using temperature data derived from satellites such as SABER (Sounding of the Atmosphere using Broadband Emission Radiometry; Garcia et al., 2005; Ern et al., 2008; Ern and Preusse, 2009), HIRDLS (High Resolution Dynamics Limb Sounder; Alexander and Ortland, 2010) and GPS-RO (Global Positioning System Radio Occultation; Tsai et al., 2004; Randel and Wu, 2005; Venkat Ratnam et al., 2006). For example, Alexander and Ortland (2010) reported a clear seasonal cycle around $16-17 \mathrm{~km}(\sim 100 \mathrm{hPa})$ in $\mathrm{KW}$ temperature observed by HIRDLS, coinciding closely with variations in background stability. A widely used method for the KW filtering from gridded data is the space-time spectral analysis introduced by Hayashi (1982). Space-time spectral filtering assumes that the linear adiabatic theory for equatorial waves on a resting atmosphere is applicable (Gill, 1982). Filtering operates on single variable data and it has been widely used to diagnose equatorial waves in the outgoing longwave radiation (OLR, e.g. Wheeler and Kiladis, 1999) and climate model outputs (e.g. Lin et al., 2006). Based on 40-year ECMWF reanalysis (ERA-40) data, Suzuki and Shiotani (2008) found that the temperature component of KWs tends to peak at $70 \mathrm{hPa}$ while the zonal wind peaks at lower altitudes, i.e. at 100 and $150 \mathrm{hPa}$ in the eastern and western hemisphere, respectively.

On the equatorial $\beta$-plane, shallow-water linear wave theory describes the KW geopotential height $\left(h_{\mathrm{kw}}\right)$ and zonal wind $\left(u_{\mathrm{kw}}\right)$ perturbations propagating zonally with phase speed $c$ as the following (Matsuno, 1966):

$h_{\mathrm{kw}}(x, y)=\frac{c}{g} u_{\mathrm{kw}}$, where

$u_{\mathrm{kW}}(x, y)=u_{0} \exp \left(-\frac{\beta y^{2}}{2 c}\right) \cos k(x-c t)$.

Here, $u_{0}$ is the zonal wind amplitude at the equator, $g$ is gravity, $y$ is the distance from the equator and $\beta=\mathrm{d} f / \mathrm{d} y$, $f$ being the Coriolis parameter. The dispersion relationship between the wave frequency $v$ and the zonal wavenumber $k$ is $v=k c$. The gravity wave speed in a layer of homogeneous fluid with mean depth $D$ is given by $c=\sqrt{g D}$ (Gill, 1982).

The KW $e$-folding decay width $a_{\mathrm{e}}$, known as the equatorial radius of deformation, is given by $a_{\mathrm{e}}=(c / 2 \beta)^{1 / 2}$. By prescribing $D$, the horizontal structure of $\mathrm{KW}$ is defined by Eq. (1) for any $k$ and can be used to simultaneously analyse wind and geopotential height perturbations due to KWs on a single horizontal level. Such analysis was carried out by Tindall et al. (2006) for the lower stratosphere for the ERA-15 data in the 1981-1993 period. Their results suggested that KWs contribute approximately $1 \mathrm{~K}^{2}$ to the temperature variance on the equator with peak activity occurring during solstice seasons at $100 \mathrm{hPa}$, during December-February at 70 and at $50 \mathrm{hPa}$ it occurs during the easterly to westerly QBO phase transition. Yang et al. (2003) used $a_{\mathrm{e}}$ as the fitting parameter for the projection of the ERA-15 data on the meridional structure of the KW and other equatorial waves. They found that the best fit trapping scale within $20^{\circ} \mathrm{N}-20^{\circ} \mathrm{S}$ is around $6^{\circ}$. The multivariate projection of data on the horizontal structures of equatorial waves including KWs on the equatorial $\beta$-plane was performed also for the short-range forecast errors of the ECMWF model (Žagar et al., 2005, 2007). For example, Žagar et al. (2007) found that forecast errors within the belt $20^{\circ} \mathrm{N}-20^{\circ} \mathrm{S}$ project onto KWs significantly more in the easterly QBO phase than in the westerly phase.

In this paper we extend the linear $\mathrm{KW}$ analysis based on the shallow-water equation theory on the equatorial $\beta$ plane to the sphere. Second, we extend the KW filtering on individual horizontal levels or vertical planes to the threedimensional (3-D) KW analysis simultaneously in wind and temperature fields. This study thus explores seasonal variability in KWs in the TTL in a multivariate fashion using most of the information on the vertical wave structure available in recent operational ECMWF analyses.

On the spherical Earth, the Kelvin mode is the slowest eastward-propagating eigensolution of the shallow-water equations (or Laplace tidal equations) linearized around a state of rest (e.g. Kasahara, 1976). In the continuously stratified atmosphere, the depth $D$ becomes the "equivalent depth" of a given baroclinic mode and we need to solve Laplace tidal equations for a range of $D$ from large (corresponding to the barotropic structure) to rather small (for high baroclinic modes) in order to consider the spectrum of KWs (e.g. Boyd, 2018). In contrast to the KW trapping on the equatorial $\beta$ plane, which is controlled by $a_{\mathrm{e}}$, i.e. by the equivalent depth, the degree of the KW equatorial confinement on the spherical Earth is in addition controlled by the zonal wavenumber (Boyd and Zhou, 2008). As shown by Boyd and Zhou (2008), even barotropic KW with $D$ around $10 \mathrm{~km}$ are on the sphere, confined within the tropical belt.

In Sect. 2 we present a methodology which diagnosis 3-D KWs in spherical datasets. Section 3 presents the KW energetics in wavenumber space focusing on the seasonal cycle. Section 4 presents seasonal KW variability in several frequency bands both for the horizontal as well as for the vertical projection KW response. Conclusions and outlook are given in Sect. 5. 


\section{Data and methodology}

The KWs are filtered using the normal-mode function (NMF) decomposition derived by Kasahara and Puri (1981) and formulated as the MODES software package by Žagar et al. (2015). Here the methodology is briefly summarized followed by the method for the computation of the KW temperature perturbations and by examples of the 3-D KW structure in global data.

Input ECMWF operational analyses cover approximately 6.5 years from January 2007 until June 2013. The dataset starts after two important updates in the ECMWF assimilation cycle: a resolution update on 1 February 2006 and the introduction of GPS-RO temperature profiles in the assimilation on 12 December 2006. The data ends at the next update in vertical resolution from L91 to L137 on 25 June 2013. The data horizontal resolution is $256 \times 128$ points in the zonal and meridional directions (regular Gaussian grid N64), respectively, on 91 irregularly spaced hybrid model levels up to around $0.01 \mathrm{hPa}$ (around $80 \mathrm{~km}$ ). The temporal resolution is 6 h, i.e. 4 times per day at 00:00, 06:00, 12:00, and 18:00 UTC. A case study of the large-scale KW in July 2007 in this dataset by Žagar et al. (2009) showed that the NMF method provides information on the 3-D wave structure and its vertical propagation in the stratosphere. Another case study from the same month demonstrated how the vertical KW structure improves as the number of vertical levels increased (Žagar et al., 2012).

\subsection{Filtering of KWs by 3-D normal-mode function expansion}

The basic assumption behind the NMF expansion is that a global state of the atmosphere described by its mass and wind variables at any time can be considered as a superposition of the linear wave solutions upon a predefined background state. The NMF decomposition derived by Kasahara and Puri (1981) uses the $\sigma$ vertical coordinate and linearization around the state of rest and realistic vertical temperature and stability stratification. The 3-D wave solutions of linearized primitive equations are represented as a truncated time series of the Hough harmonic oscillations and the vertical structure functions. The assumption of separability leads to separate equations for the vertical structure and horizontal oscillations. The latter are known as shallow-water equations on the sphere or Laplace tidal equations without forcing. The two systems are coupled by a separation parameter $D$ which is called the equivalent height (Boyd, 2018). Eigenmodes of the global shallow-water equations are known as Hough harmonics. They describe two types of wave motions: Rossby waves and inertio-gravity waves which obey their corresponding dispersion relationships on the spherical Earth.
The expansion of a global input data vector $\boldsymbol{X}(\lambda, \varphi, \sigma)=$ $(u, v, h)^{T}$ can be represented by a discrete finite series as

$\left|\begin{array}{c}u(\lambda, \varphi, \sigma) \\ v(\lambda, \varphi, \sigma) \\ h(\lambda, \varphi, \sigma)\end{array}\right|=\sum_{m=1}^{M} \mathbf{S}_{\mathrm{m}}\left[\sum_{n=1}^{R} \sum_{k=-K}^{K} \chi_{\mathrm{n}}^{k}(m) \mathbf{H}_{\mathrm{n}}^{k}(\lambda, \varphi ; m)\right] G_{m}(\sigma)$.

The input data vector contains wind components $u, v$ and the transformed geopotential height $h$ defined as $h=$ $g^{-1} P$, where $g$ is the gravity and $P$ is defined as $P=$ $\Phi+R T_{0} \ln \left(p_{\mathrm{s}}\right)$; that is, it is the sum of geopotential $\Phi$ and a surface pressure, $p_{\mathrm{s}}$, term. Two other variables represent the specific gas constant for dry air $(R)$ and the globallyaveraged vertical temperature profile $\left(T_{0}(\sigma)\right)$. The zonal and vertical truncations ( $K$ and $M$, respectively) define maximal numbers of zonal waves at a single latitude (wavenumber $k$ ) and a maximal number of vertical modes (denoted $m)$, respectively. For every vertical structure eigenfunctions $G_{m}(\sigma)$, Hough harmonic functions, $\mathbf{H}_{\mathrm{n}}^{k}(\lambda, \varphi)$, describe nondimensional oscillations in the horizontal plane of the fluid with the mean depth equal the equivalent depth $D_{\mathrm{m}}$. The parameter $D_{\mathrm{m}}$ appears in Eq. (2) in the diagonal matrix $\mathbf{S}_{\mathrm{m}}$ with elements $\left(g D_{\mathrm{m}}\right)^{1 / 2},\left(g D_{\mathrm{m}}\right)^{1 / 2}$ and $D_{\mathrm{m}}$, which normalizes the input data vector after the vertical projection and thereby removes dimensions. Parameter $R$ is the total number of meridional modes which is a sum of the eastward inertiogravity waves (EIG), westward inertio-gravity waves (WIG) and Rossby waves. Linearization about the state of rest is not a drawback of the method as wave frequencies are used solely for the formulation of the projection basis and not for studying wave propagation properties. As shown by Kasahara (1980) (see also its Corrigendum) the meridional structures of the Hough functions for large scales are not significantly different if the linearization is performed around the non-zero mean zonal flow. The impact of latitudinal shear on the KWs was shown to be negligible by Boyd (1978). Further details of the NMF projection procedure are given in Žagar et al. (2015).

For each zonal wavenumber, the Kelvin mode is the lowest eastward-propagating latitudinal Hough function. In Eq. (2), the $\mathrm{KW}$ is represented by the non-dimensional complex expansion coefficients $\chi_{\mathrm{n}}^{k}(m)$ with the meridional index $n=$ 1. However, to follow often-used notation, we shall denote the $\mathrm{KW}$ in the remainder of this study as the $n=0 \mathrm{EIG}$ mode, i.e. the $\mathrm{KW}$ wind and geopotential height are represented by coefficients $\chi_{\mathrm{kw}}=\chi_{0}^{k}(m)$. The truncation values are $K=85$ and $M=60$. This means that the KW signal in 3-D circulation at a single time instant consists of 5100 waves, 85 waves in every shallow-water equation system. Higher vertical modes were left out as their equivalent depth is smaller than $2 \mathrm{~m}$ and their contribution to the total $\mathrm{KW}$ signal is negligible in the outputs in the TTL and the stratosphere. The relation between the truncation parameters and the normal-mode projection quality is discussed in Žagar et al. (2015) and references therein.

Once the forward projection is carried out and coefficients $\chi_{\mathrm{n}}^{k}(m)$ are produced, filtering of KWs in physical space can 
be performed through Eq. (2) after setting all $\chi$, except those representing the KWs, to zero. The result of filtering are fields $u_{\mathrm{kw}}, v_{\mathrm{kw}}$ and $h_{\mathrm{kw}}$, which provide the KW zonal wind, meridional wind and geopotential height perturbations. Notice here that in contrast to the equatorial $\beta$-plane, KWs on the sphere have a small meridional wind component which is thus left out from the discussion (Boyd, 2018).

The KW temperature perturbation, $T_{\mathrm{kw}}$, can be derived from the $h_{\mathrm{kw}}$ fields on $\sigma$ levels using the hydrostatic relation in $\sigma$ coordinates:

$T_{\mathrm{kw}}=-\frac{g \sigma}{R} \frac{\partial h_{\mathrm{kw}}}{\partial \sigma}$.

The orthogonality of the normal-mode basis functions provides KW energy as a function of the zonal wavenumber and vertical mode. After the forward projection, the energy spectrum of total (potential and kinetic) energy for each KW can be computed using the energy product for the $k$ th and $m$ th normal modes (Žagar et al., 2015) as

$I_{\mathrm{kw}}(k, m)=\frac{1}{2} g D_{\mathrm{m}} \chi_{\mathrm{kw}}\left[\chi_{\mathrm{kw}}\right]^{*}$.

The units are $\mathrm{J} \mathrm{kg}^{-1}$. The $\mathrm{KW}$ global energy spectrum as a function of the zonal wavenumber is obtained by summing energy in all vertical modes:

$I_{\mathrm{kw}}(k)=\frac{1}{2} \sum_{m=1}^{M} g D_{\mathrm{m}} \chi_{\mathrm{kw}}\left[\chi_{\mathrm{kw}}\right]^{*}$.

\subsection{Examples of 3-D structure of KWs in L91 analyses}

KWs are shown in Figs. 1-2 for a few days in July 2010 to introduce and illustrate their properties as filtered by the NMF methodology.

Figure 1 illustrates the meridional structure of KWs on 25 July 2010 on 2 levels. KW activity was found largest in the zonal wind component at $150 \mathrm{hPa}$ over the Indian Ocean. The geopotential dipole structure is centred over the convective hotspot over the Maritime Continent. At $100 \mathrm{hPa}$, we find the largest amplitude of $\mathrm{KW}$ temperature perturbations up to $4 \mathrm{~K}$ positioned above the zonal wind maxima at $150 \mathrm{hPa}$. The meridional wind component of the KW is non-zero in spherical coordinates, but is at most $0.22 \mathrm{~m} \mathrm{~s}^{-1}$ at $100 \mathrm{hPa}$, which is negligible compared to the zonal wind component (maximum $12.5 \mathrm{~m} \mathrm{~s}^{-1}$ ) making the $\mathrm{KW}$ wind field primarily zonal. Note that the presented horizontal structure at a single level is a superposition of 60 vertical modes, i.e. 60 shallow water models with equivalent depths from about $10 \mathrm{~km}$ to a couple of metres.

Figure 2 illustrates day-to-day filtered $\mathrm{KW}$ fields along the equator on three separate July days in 2010, namely 25,28 and 31. Both zonal wind (blue-to-red shades) and temperature fields (red contours) are shown. Without any predefined constrains on the $\mathrm{KW}$ propagation, one can observe a rich variety of $\mathrm{KW}$ behaviour occurring in time: from the quasistationary dipole patterns centred at $160 \mathrm{hPa}$ to a wave package of free-propagating wave structures in the stratosphere transiting from the western into the eastern hemisphere.

In the stratosphere, the uppermost easterly wind component in blue shades around 30-50 hPa moves in eastward and downward directions, demonstrating the upward transport of KW energy (Andrews et al., 1987). KW amplitudes were largest over the eastern hemisphere with temperatures up to $4 \mathrm{~K}$ and zonal winds up to $12 \mathrm{~m} \mathrm{~s}^{-1}$. The large amount of KW activity occurred during the easterly phase of the QBO with strong easterly winds present between 30 and $80 \mathrm{hPa}$ (not shown), providing favourable conditions for strong KW activity.

Between 100 and $200 \mathrm{hPa}$ during the second half of July, there was low-frequency KW activity present in the form of a stationary and robust "wave-1" pattern with strong KW easterly winds up to $24 \mathrm{~m} \mathrm{~s}^{-1}$ in the eastern hemisphere and KW westerly winds up to $10 \mathrm{~m} \mathrm{~s}^{-1}$ in the western hemisphere. The high vertical resolution within the TTL resolves shallow KW structures and a typical slanted structure towards the east in KW easterlies as well. The appearance and strength of horizontal $\mathrm{KW}$ response coincides with the presence of strong easterly winds in the TTL in the eastern hemisphere during this period (not shown). Figure 2 also shows that below $300 \mathrm{hPa}$ the KW activity decreases and we shall not discuss levels under $300 \mathrm{hPa}$ in this paper.

The zonal wind and temperature components are coupled through Eq. (3), which states that the amplitude of the negative KW temperature perturbation is proportional to the negative vertical gradient in geopotential (and vice versa), as well as in the zonal wind since the zonal wind and geopotential are in phase. Horizontally, the cold anomaly is always located between the westerly and the easterly phase of the zonal wind component. Vertically, maximal positive temperatures are observed between easterly winds below and westerly winds above. An estimate of the vertical wavelength can be made based on alternating zonal wind minima and maxima. For example, on 25 July a well-developed KW package extending into the stratosphere moved from the western into the eastern hemisphere. A quasi-stationary component of the wave package is observed around $60^{\circ} \mathrm{E}$ with easterly winds located at $50(\sim 21.5 \mathrm{~km})$ and $150 \mathrm{hPa}(\sim 13.5 \mathrm{~km})$, implying a vertical wavelength of around $8 \mathrm{~km}$.

More examples based on a daily basis filtered data from the 10-day deterministic forecast of the ECMWF can be found on the MODES website ${ }^{1}$.

\subsection{Other data and impact of the background state}

In addition to the outputs from modal decomposition, full zonal wind and temperature fields from ECMWF analyses are used to compute the background fields based on the

\footnotetext{
${ }^{1}$ http://meteo.fmf.uni-lj.si/MODES/, last access: 12 June 2018
} 


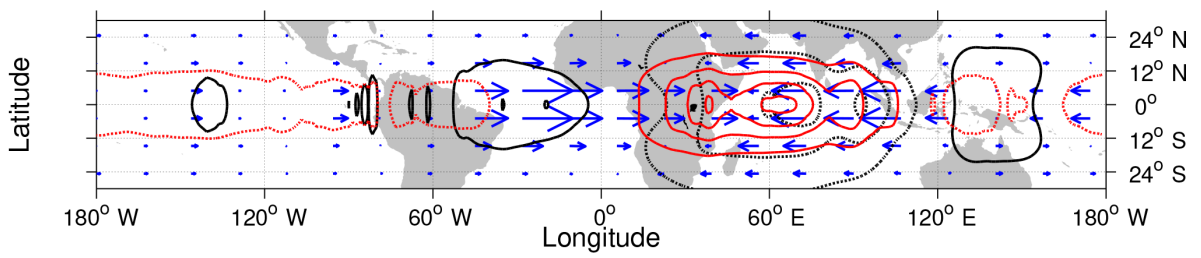

(a) 25-7-2010, level $38(\sim 100 \mathrm{hPa})$

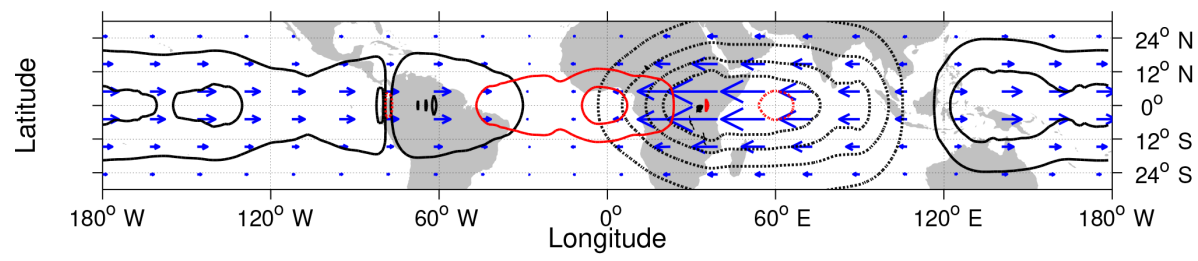

(b) 25-7-2010, level $44(\sim 150 \mathrm{hPa})$

Figure 1. The horizontal structure of KWs in the ECMWF analysis data on 25 July 2010 at (a) 100 and (b) $150 \mathrm{hPa}$. The geopotential height perturbations $\left(h_{\mathrm{kw}}\right)$ are shown by black contours, every $20 \mathrm{~m}$, whereas temperature perturbations $\left(T_{\mathrm{kw}}\right)$ are coloured red (every $\left.1 \mathrm{~K}\right)$. Dashed contours represent negative and full line contour positive perturbations. Zero lines are omitted.

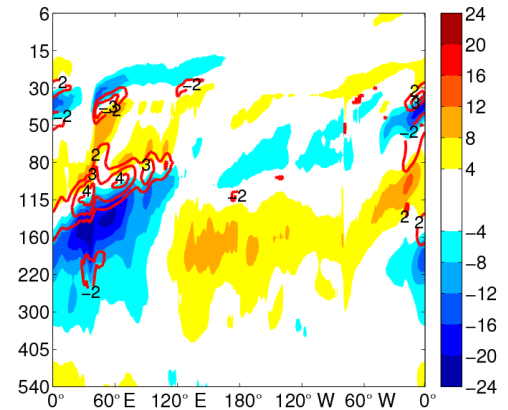

(a) 25 July 2010

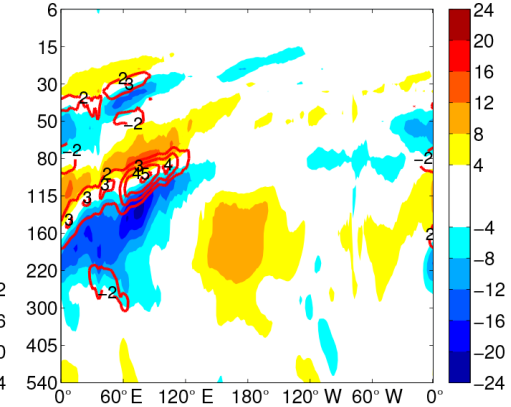

(b) 28 July 2010

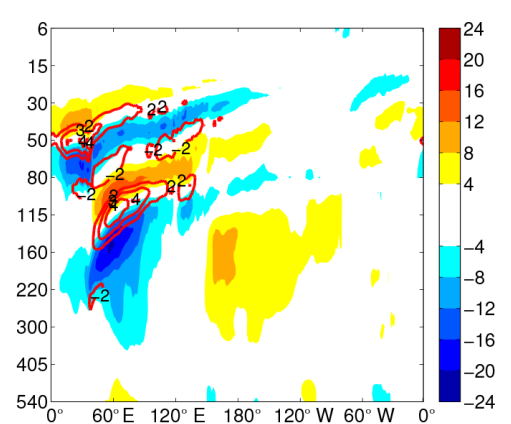

(c) 31 July 2010

Figure 2. Longitude-pressure cross section of the Kelvin wave (KW) zonal wind (red-blue shaded contours) and temperature (red contours) perturbations along $0.7^{\circ} \mathrm{N}$ on (a) 25 July, (b) 28 July and (c) 31 July 2010 . Temperature is shown every $1 \mathrm{~K}$, starting at $2 \mathrm{~K}$. Zonal winds are drawn every $4 \mathrm{~m} \mathrm{~s}^{-1}$. Zero lines are omitted.

same N64 grid and over the same period (January 2007-June 2013). Zonal wind $U$ and static stability $N$ are latitudinally averaged in the belt $5^{\circ} \mathrm{S}-5^{\circ} \mathrm{N}$ on all model levels to produce their zonal structure.

Static stability profiles are estimated through

$N^{2}=\frac{g^{2}}{\Theta} \frac{\partial \Theta}{\partial \phi}$

in units of $\mathrm{s}^{-2}$ and are defined on hybrid model levels on which the geopotential field $\phi$ and the potential temperature field $\Theta$ are derived a priori from the input data. Both fields are shown in Fig. 3.

The zonal wind field has the largest values on average in the TTL around $150 \mathrm{hPa}$ with westerly winds peaking in the western hemisphere over the Pacific Ocean and easterly winds peaking in the eastern hemisphere over the Indian Ocean and Indonesia. It represents a typical timeaveraged outflow pattern in response to tropical convection (e.g. Fueglistaler et al., 2009). Throughout the seasons there is a longitudinal shift of this pattern following the convective source which is most clearly observed at $150 \mathrm{hPa}$. Such a seasonal shift is visible up to $100 \mathrm{hPa}$ in Fig. $3 \mathrm{~b}$ where winds are weaker compared to $150 \mathrm{hPa}$. In northern winter, zonal winds are strongest over Indonesia and the eastern Pacific with the zonal wind maxima position and strength similar compared to the longer ERA-40 dataset used by Suzuki and Shiotani (2008). During boreal summer, easterly winds mainly prevail over the Indian Ocean, which is linked to the Indian monsoon season.

At $100 \mathrm{hPa}$, the static stability illustrates the strongest seasonal cycle with values ranging from near-tropospheric values of $3 \times 10^{-4} \mathrm{~m} \mathrm{~s}^{-2}$ during northern winter towards stratospheric values of $5-6 \times 10^{-4} \mathrm{~m} \mathrm{~s}^{-2}$ during boreal summer. Note also the resolved local maxima in static stability at $80 \mathrm{hPa}$ above the warm pools, known as the tropical inversion layer (TIL) and which is possibly wave-driven (Grise et al., 


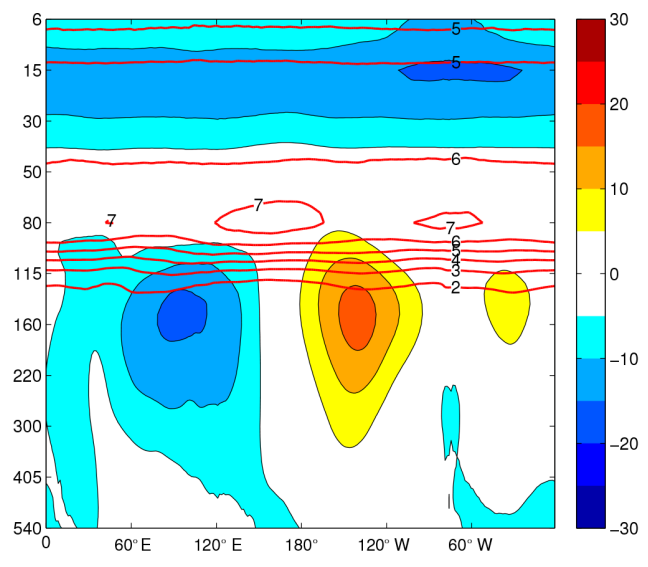

(a) Longitude-height, 6-year average

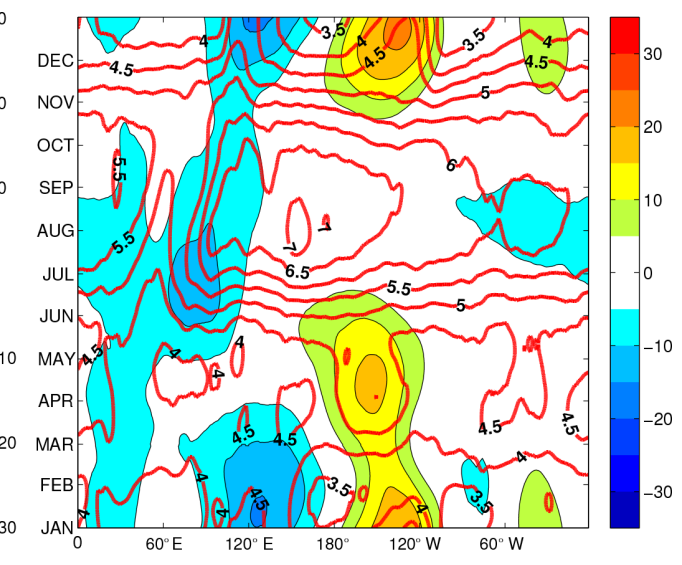

(b) Longitude-time at $100 \mathrm{hPa}$, 6-year average

Figure 3. Six-year average of the zonal wind and static stability fields of the ECMWF operational analyses. Both fields are latitudinally averaged over $5^{\circ} \mathrm{S}-5^{\circ} \mathrm{N}$, and have been low-pass filtered a priori with a cut-off period of 90 days to highlight seasonal variability. (a) Longitudeheight section and (b) longitude-time section at $100 \mathrm{hPa}$. Zonal winds are coloured by blue-to-red contours, each $5 \mathrm{~m} \mathrm{~s}^{-1}$, whereas and static stability is shown in red contours, each (a) $1 \times 10^{-4} \mathrm{~s}^{-2}$ and (b) $0.5 \times 10^{-4} \mathrm{~s}^{-2}$. Zero lines are omitted.

2010; Kedzierski et al., 2016). Figure 3b suggests that the TIL descends down to $100 \mathrm{hPa}$ during boreal summer months peaking over the western Pacific, in agreement with the cycle found in GPS-RO observations by Grise et al. (2010).

KWs are subject to wave modulation in changing background environments. Along its trajectory, the potential energy of the KW changes with varying background winds and stability which can be largely described by linear wave theory as long as waves are not near their critical level involving breaking and dissipation (Andrews et al., 1987). For simplification, KW modulation can be examined for the case of pure zonal as well as pure vertical wave propagation based on the wave modulation analysis performed by Ryu et al. (2008). A few key points on their local wave action conservation principle are summarized in the following.

In the tropical atmosphere, zonal modulation is the dominant process for KWs propagating in the stratosphere and in all non-easterly winds in the TTL. Vertical modulation becomes important in the presence of easterly winds within the TTL. Zonal modulation is found to affect both $u_{\mathrm{kw}}$ and $T_{\mathrm{kw}}$ components and their amplitudes are proportional to the Doppler-shifted phase speed by $(c-U)^{1 / 2}$ in the case of a pure zonal propagation direction. This means that KWs diminish in amplitude over regions with westerly winds and become more prone to dissipative processes, while amplify over regions with easterly winds ${ }^{2}$. In the case of pure vertical modulation, the change in wave potential energy mainly fluctuates with the temperature component of the KW. Along the rays' vertical path, the waves amplitude is proportional to the Brunt-Väisälä frequency as $\propto N^{3 / 2}$, and to the Doppler-

\footnotetext{
${ }^{2}$ Keeping in mind that vertical wave propagation and consequently modulation becomes increasingly important as well wherever easterly winds are strong.
}

shifted phase speed as $\propto(c-U)^{-1 / 2}$, such that $N$ is expected to play a primary role above $120 \mathrm{hPa}$ where its value starts increasing rapidly (see Fig. 3).

Alexander and Ortland (2010) showed through wave modulation principles that temporal variations in zonal-mean $N$ indeed are correlated with observed KW amplitudes at $16 \mathrm{~km}$ (approx. $100 \mathrm{hPa}$ ). A more extensive wave modulation analysis was described by Flannaghan and Fueglistaler (2013) using the full ray-tracing equations to demonstrate that zonal winds in the TTL not only modulate KWs locally but also create a lasting modulating effect on wave activity through ray convergence in the stratosphere. In particular, the seasonal cycle of the upper tropospheric easterlies (on average located over the western Pacific), that acts as an escape window for KWs throughout the year and largely explains the longitudinal structure of KW zonal wind and temperature climatology.

We shall present the seasonal variability in tropical convection by using the OLR dataset with daily outputs from the NOAA Interpolated OLR product (Liebmann and Smith, 1996). The OLR product, often used as a proxy for convection, is extracted on a $2.5^{\circ} \times 2.5^{\circ}$ grid and interpolated on a N64 grid. Latitudinal averages are derived over a larger domain, namely over $15^{\circ} \mathrm{S}-15^{\circ} \mathrm{N}$ since organized convection tends to happen more remote from the equator, especially during the summer monsoon season over the Asian continent.

\section{KW energetics}

We start with a discussion of the KW energy distribution among zonal wavenumbers as given by Eq. (5), followed by seasonal differences. 


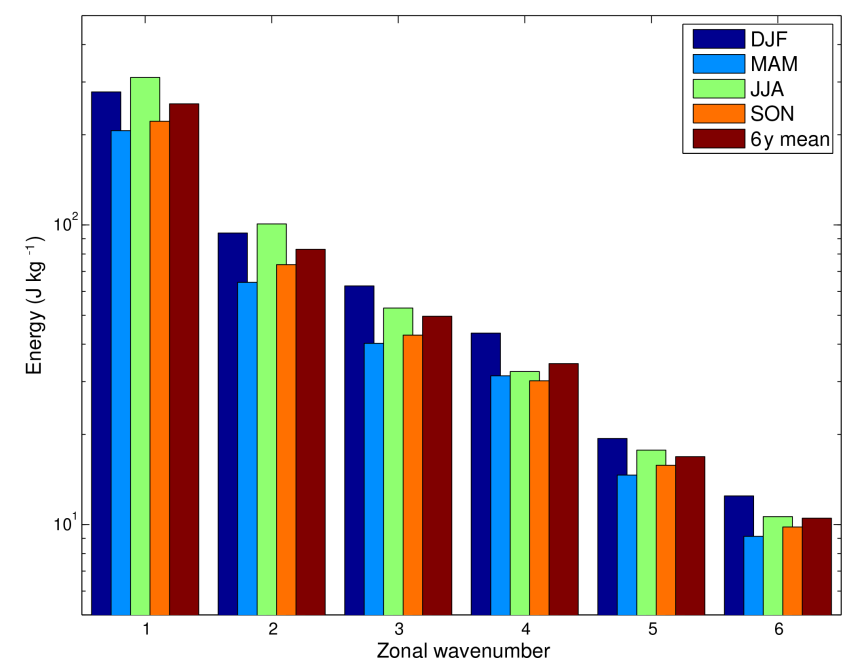

Figure 4. Kelvin wave (KW) energy (in $\mathrm{J} \mathrm{kg}^{-1}$ ) as a function of the zonal wavenumber $k$ for $k=1$ to 6 . For each $k$, seasonal averages are shown along with the total average as described by the legend. Energy is vertically integrated over 60 vertical modes. Further details are in Sect. 3.1.

\subsection{Energy distribution of KWs}

The seasonal cycle in the energy-zonal-wavenumber spectra is shown in Fig. 4 after summing up over all vertical modes. On average, energy decreases as the zonal wavenumber increases as typical for atmospheric energy spectra. As we deal with large scales, we show only the first 6 zonal wavenumbers with energy values shown separately for the annual mean and the four seasons separately.

Figure 4 shows that largest seasonal variations in $\mathrm{KW}$ energy are found at the largest zonal scales. For all zonal wavenumbers, above annual-mean energy values are observed during DJF and JJA seasons while SON and MAM are below annual-mean energy. In the zonal wavenumber 1, total KW energy varies between $200 \mathrm{~J} \mathrm{~kg}^{-1}$ in MAM season and somewhat over $300 \mathrm{~J} \mathrm{~kg}^{-1}$ in JJA. In wavenumber 2, values do not exceed $100 \mathrm{~J} \mathrm{~kg}^{-1}$ and JJA still contains the largest energy. At higher wavenumbers, DJF season becomes the most energetic. In $k>4$, total $\mathrm{KW}$ energy is under $20 \mathrm{~J} \mathrm{~kg}^{-1}$ and continue to reduce with $k$. The slope of the KW energy spectrum is between $-5 / 3$ and -1 at planetary scales (not shown), similar to the spectra presented in Žagar et al. (2009) for July 2007 data. The JJA spectra has on average the steepest slope compared to other seasons, in particular the DJF spectra. The energy distribution on planetary scales is mainly associated with large-scale tropical circulation established in response to ongoing tropical convection. Therefore, the zonal distribution of tropical convection may likely play a crucial role in explaining DJF and JJA season differences of KW energy, which will be explored in the next section.

\subsection{Seasonal cycle of KW energy}

Figure 5 illustrates more details on the seasonal cycle by showing KW energy-time series at the largest scales represented by zonal wavenumbers $k=1, k=2$ and remaining scales $k>2$. During most JJA seasons and occasionally in DJF (e.g. 2008) the total amount of KW energy in $k=1$ can reach up to $600 \mathrm{~J} \mathrm{~kg}^{-1}$, or twice the JJA average. The minimum in $k=1 \mathrm{KW}$ energy mainly occurs during October followed by April with values dropping towards $100 \mathrm{~J} \mathrm{~kg}^{-1}$, or half the SON average. The temporal pattern in $k=2$ is similar to the $k=1$ pattern, but with a less pronounced semiannual cycle with maximum values up to $200 \mathrm{~J} \mathrm{~kg}^{-1}$ and minimum values towards $30 \mathrm{~J} \mathrm{~kg}^{-1}$. On zonal scales $k>2$, KWs still show a semiannual cycle with highest vertically integrated values of energy in DJF.

In particular, for zonal wavenumber $k=1$ one can distinguish inter-monthly in addition to semiannual variability. Inter-monthly variability is most clearly observed during JJA, for example in July 2011 where one can distinguish six separate peaks of over $400 \mathrm{~J} \mathrm{~kg}^{-1}$ energy over a period of approximately 90 days resembling an average wave period of about 18 days. These are typical periods for free-propagating KWs as observed in the TTL and lower stratosphere (e.g. Randel and $\mathrm{Wu}, 2005)$. Note here again that our KW energy is vertically integrated over the whole model depth. This means that the observed inter-monthly variability in KWs appears to be dominated by the cyclic process of free-propagating KWs entering the TTL, amplifying due to changing environmental conditions, followed by wave breaking or dissipation.

The dominant scales of temporal variability in KWs are illustrated by a frequency spectrum of $k=1$ in Fig. 6. The spectrum is produced by the Fourier transform of energy time series of 6.5 years. The resulting power spectrum has been smoothed by taking the Gaussian-shaped moving averages over the raw spectrum by using the Daniell kernel three times (Shumway and Stoffer, 2010). The spectrum contains a peak at 1 day period associated with the diurnal tide partially projecting on the KWs. After that, a gradual increase in energy is seen towards the 16-day period with multiple individual periods standing out. For periods longer than 20 days, individual peaks are found close to 25, 43 and 59 days. After that, most $\mathrm{KW}$ energy is contained by far in the semiannual cycle. The frequency spectrum provides a useful starting point for the discussion in the next section when the spatiotemporal patterns of KWs shall be examined in several spectral domains.

Returning to Fig. 5, a low-pass filter with 90-day cut off has been applied on KW energy in order to keep only the two main spectral peaks in Fig. 6. The result is visible as the thicker black line in Fig. 5 for all three zonal wavenumber groups. A semiannual cycle for all zonal wavenumbers is evident with most energy observed around January and July, while least energy is observed approximately one month after the equinoxes. During the years 2007, 2010, 2011 and 2012, more $k=1 \mathrm{KW}$ energy is observed during JJA compared to 


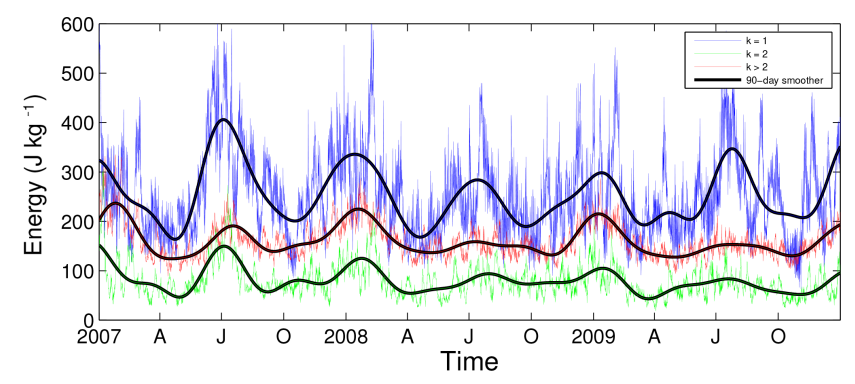

(a) 2007-2009

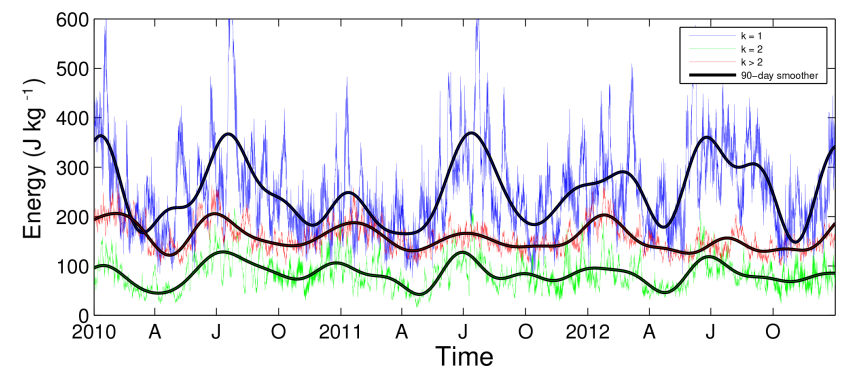

(b) $2010-2012$

Figure 5. Time series of the global total KW energy for various zonal wavenumbers over the following periods: (a) 2007-2009 and (b) 2010-2012. Labels on the $x$ axis "A", "J" and "O" refer to the first days of April, July and October, respectively. Presented are the zonal wavenumbers $k=1$ (blue line), $k=2$ (green line) and all smaller zonal scales, $k>2$ (red line). A 90 day low-pass filter has been applied (black lines) for each time series in order to filter out high-frequency variability and to highlight seasonal variability.

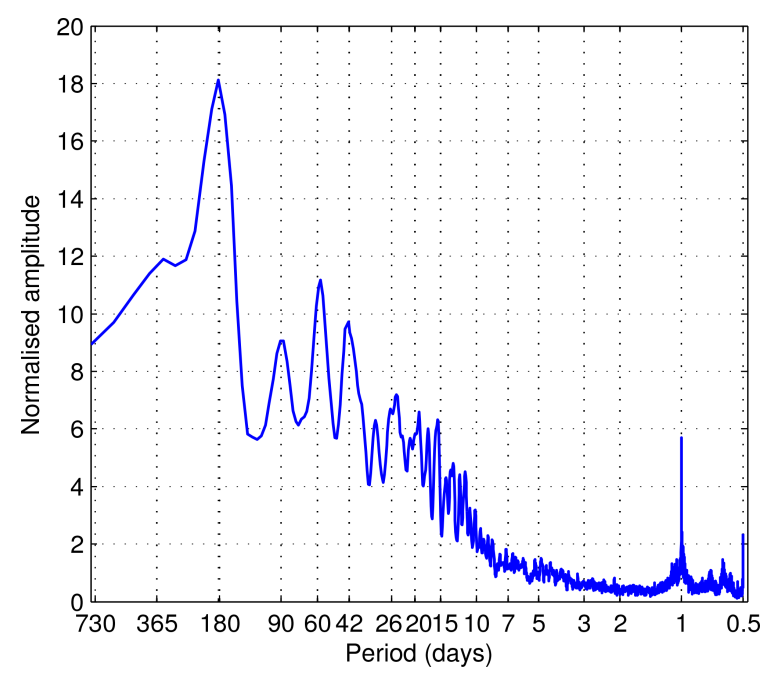

Figure 6. Kelvin wave $(\mathrm{KW})$ frequency spectrum for the zonal wavenumber $k=1$. The 1-2-1 filter with a Daniell kernel has been used to smooth the initial raw power spectra.

the follow-up DJF season. The DJF of 2009-2010 was, for example, above average with energy values for $k=1$ above $350 \mathrm{~J} \mathrm{~kg}^{-1}$.
The year-to-year differences can be explained by many coupled factors. In general, one expects the vertically integrated KW activity to increase when background wind conditions become favourable, i.e. in the presence of easterly winds. This occurs in the TTL in relation to strong convective outflow (Garcia and Salby, 1987; Suzuki and Shiotani, 2008; Ryu et al., 2008; Flannaghan and Fueglistaler, 2013) during DJF and JJA seasons mainly. Moreover, KW activity is enhanced whenever easterly QBO winds are present down into the lower stratosphere (Baldwin and Coauthors, 2001; Alexander and Ortland, 2010) or during El Niño (Yang and Hoskins, 2013). The latter factor may partly explain a large difference in the KW energy during the El Niño DJF of 2009-2010 and the below-average energy level a year after, during the strong La Niña DJF period of 2010-2011. However, during the La Niña DJF of 2007-2008, the amount of $\mathrm{KW}$ energy is above normal. That season, however, was characterized by above-normal Madden-Julian Oscillation activity which often occurs during favourable easterly QBO conditions in the stratosphere (Son et al., 2017). During 2010 2011, DJF season stratospheric winds were largely westerly thereby prohibiting KW activity. The role of these lowfrequency atmospheric phenomena on $\mathrm{KW}$ seasonal variability is a topic of further research.

Finally, Fig. 5 also shows that KW activity in July 2007, previously examined by Žagar et al. (2009), was exceptionally strong. A large part of that energy, (somewhat more than half) belonged to zonal wavenumber 1 . In spatiotemporal terms, it is associated with the presence of a strong dipole structure in the TTL (as in Fig. 2), which is colocated with favourable easterly wind conditions in the TTL as well as in the stratosphere (not shown). In fact, at $50 \mathrm{hPa}$ the QBO was just at the beginning of its easterly phase in July 2007.

\section{A spatiotemporal view on KW seasonal variability}

\subsection{KW decomposition among wave periods}

In this section, the spatiotemporal view of KWs shall be presented over three dominant ranges of wave periods in Fig. 6, namely (i) the (semi)annual cycle using a low-pass filter with cut-off period at 90 days, (ii) the intra-seasonal period using a bandpass filter over periods between 20 and 90 days and finally (iii) the intra-monthly period with bandpass filtered periods between 3 and 20 days. The chosen periods, especially the intra-monthly periods, are similar to those used in previous studies. In each case, mean 6-year fields as well as seasonal means shall be presented.

Note that our temporal filtering operates on time series of KW signals at every grid point. This is different from the commonly applied space-time filtering following Hayashi (1982) that applies KW dispersion relations. Our filtered KWs can appear stationary or even westward-shifted due 
to westward-moving sources of the KW amplification (e.g. easterly winds, high static stability in the TTL).

Both KW components $u_{\mathrm{kw}}$ and $T_{\mathrm{kw}}$ are Fouriertransformed to frequency space where the spectral expansion coefficients $\chi_{\mathrm{kw}}$ in domains outside the desired frequency ranges are put to zero. Case (i) results in $\mathrm{KW}$ components $u_{\mathrm{kw}, 1}$ and $T_{\mathrm{kw}, 1}$ where 1 indicates the low-frequency component. Case (ii) results in $u_{\mathrm{kw}, \mathrm{m}}$ and $T_{\mathrm{kw}, \mathrm{m}}$ where $\mathrm{m}$ indicates the intra-monthly period. Case (iii) results in fields $u_{\mathrm{kw}, \mathrm{h}}$ and $T_{\mathrm{kw}, \mathrm{h}}$ where $\mathrm{h}$ stands for the high-frequency component. Previous studies have defined free-propagating KWs over similar ranges (3-20 days, Alexander and Ortland, 2010; 423 days, Suzuki and Shiotani, 2008) and similarly for intraseasonal periods (23-92 days, Suzuki and Shiotani, 2008). Next, seasonal averages will be taken over the four seasons, resulting in variables ${\overline{u_{\mathrm{kw}, 1}}}^{s}$ and ${\overline{T_{\mathrm{kw}, 1}}}_{\mathrm{s}}$ for the low-frequency component and similarly for the other two cases. The superscript $s$ represents one of the four seasons: northern winter $(s=\mathrm{DJF})$, spring $(s=\mathrm{MAM})$, summer $(s=\mathrm{JJA})$ and autumn $(s=\mathrm{SON})$.

Cases (ii) and (iii) contain purely subseasonal variability and therefore one can expect their 6-year means to be zero-valued since variability beyond 90 days has been put to zero. Similarly, mean fields for each of the four seasons result in ${\overline{u_{\mathrm{kw}, \mathrm{h}}}}^{s} \ll{\overline{u_{\mathrm{kw}, 1}}}^{s}$ and ${\overline{u_{\mathrm{kw}, \mathrm{m}}}}^{s} \ll{\overline{u_{\mathrm{kw}, 1}}}^{s}$ and the same for the temperature component. This reflects the fact that positive and negative phases of the fast $\mathrm{KW}$ responses average out to approximately zero on seasonal timescales (figure not shown). Therefore, the seasonal mean of the absolute amplitudes of the zonal wind and temperature are examined instead, i.e. $\overline{\left|u_{\mathrm{kw}, \mathrm{h}}\right|}$ and $\overline{\left|u_{\mathrm{kw}, \mathrm{m}}\right|}$ and similarly for temperature. This describes seasonal fluctuations in subseasonal KW amplitudes $^{3}$.

Figure 7 shows results for all three cases after taking means over the whole period. The left panel resembles a dominant "wave-1" structure with zonal wind maximized around $140 \mathrm{hPa}$. Easterly $\mathrm{KW}$ winds are strongest around $60^{\circ} \mathrm{E}$ and westerly winds around the International Date Line. Note that two stationary perturbations over African $\left(30^{\circ} \mathrm{E}\right)$ and South American $\left(80^{\circ} \mathrm{W}\right)$ orography are the result of our terrain-following NMF analysis. If one compares the $\mathrm{KW}$ zonal wind pattern with the climatological zonal wind pattern in Fig. 3a, it can be observed that the zonal wind pattern is located around $20^{\circ}$ west of the climatological pattern. Wave temperature perturbations are largest where the vertical gradients in zonal wind are largest, which explains the quadrupole structure. Warm and cold KW anomalies are lo-

\footnotetext{
${ }^{3}$ Most previous studies define KW activity as the square amplitude rather than absolute amplitude. In our high-resolution dataset we observe highly localized patterns of the KW activity in the eastern hemisphere due to ongoing wave amplification. By using absolute amplitudes we better visualize the longitudinal structure of the $\mathrm{KW}$ activity in comparison to its local maxima.
}

cated at $100 \mathrm{hPa}$ in the eastern and western hemispheres, respectively, and vice versa at $200-300 \mathrm{hPa}$.

The average low-frequency or seasonal KW structure has a significant resemblance with the classical Gill-type KW solution (Gill, 1980) describing a steady-state linear wave response to convective forcing. The Gill-type KW solution is characterized by westerly upper-troposphere winds east of the large-scale convective source. In response to the seasonal cycle of convection, the solution in Fig. 7a also illustrates, in addition to a low-frequency $\mathrm{KW}$ variability in westerly winds, a considerable low-frequency variability west of the convective outflow. This part of the signal represents the wave modulation effect of the propagating KWs on seasonal timescales.

The middle panel of Fig. 7 shows the average distribution of KW activity on intra-seasonal timescales. The activity is largest in the eastern hemisphere with average zonal wind maxima up to $3 \mathrm{~m} \mathrm{~s}^{-1}$ and temperature maxima up to $0.7 \mathrm{~K}$. Zonal wind activity is largest over a broad area between 90 and $150 \mathrm{hPa}$ over the Indian Ocean and the Maritime Continent. Temperature activity occurs slightly higher around $90-100 \mathrm{hPa}$. Intra-seasonal activity is locally somewhat increased also around $120^{\circ} \mathrm{W}$, west of the Andes mountain range.

Finally, Fig. 7c illustrates the average distribution of intramonthly KWs. The eastern hemisphere again makes up for the larger KW activity than the western hemisphere, but the maximum is located more upward in comparison to the intraseasonal scales, around $80 \mathrm{hPa}$. Zonal wind activity peaks up to $3 \mathrm{~m} \mathrm{~s}^{-1}$ over a broad range of $70-110 \mathrm{hPa}$ and temperature peaks over a more narrow area around $76 \mathrm{hPa}$ (up to $0.75 \mathrm{~K})$. The main area for $\mathrm{KW}$ activity is found over the Indian Ocean region, while least wave activity is above the central Pacific. Towards the stratosphere, $\mathrm{KW}$ activity reduces and becomes more uniform along the longitudinal direction.

\subsection{Low-frequency $\mathrm{KW}$ variability}

The seasonal patterns of the low-frequency components of the $\mathrm{KW}$ is presented as pressure-longitudinal cross sections along the equator (at $0.7^{\circ} \mathrm{N}$ ) of the $\mathrm{KW}$ seasonal means, given by ${\overline{\left[u_{\mathrm{kw}, 1}\right]}}^{s}$ and ${\overline{\left[T_{\mathrm{kw}, 1}\right]}}^{s}$ in Fig. 8 .

The largest amplitudes are found during the JJA months. A strong dipole "wave-1" pattern is evident in the TTL. The strongest zonal winds are found close to $150 \mathrm{hPa}$ with easterlies up to $-12 \mathrm{~m} \mathrm{~s}^{-1}$ centred over the Indian Ocean and westerlies up to $6 \mathrm{~m} \mathrm{~s}^{-1}$ over the western Pacific. Negative temperature $\mathrm{KW}$ anomalies at $110 \mathrm{hPa}$ are strongest as well during JJA with values up to $1.5 \mathrm{~K}$ over the Indian Ocean and annually averaged value of $-0.5 \mathrm{~K}$ over the western Pacific.

During DJF, the dipole pattern has shifted more eastward and upward compared to JJA and has a more slanted structure. Easterly (westerly) KW winds are located more east over the Maritime continent (central Pacific) and are centred at $130 \mathrm{hPa}$. The upper temperature dipole pattern is 


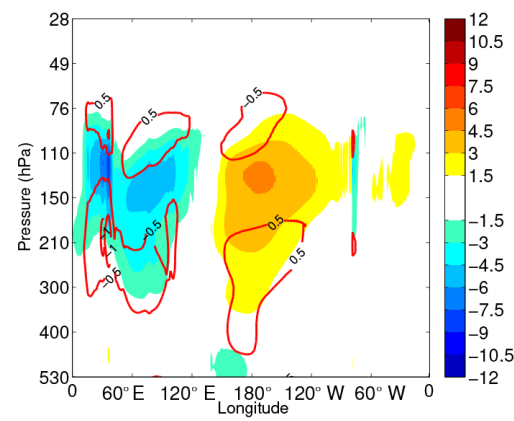

(a) $>90$ days

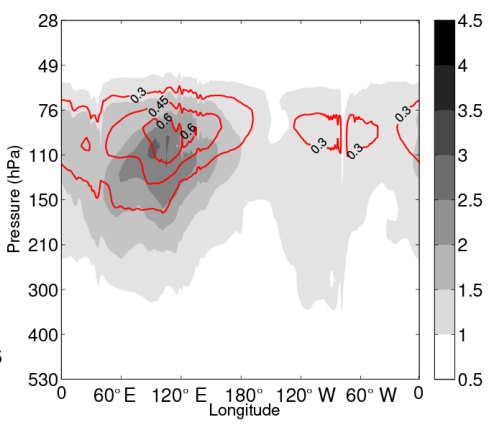

(b) $20-90$ days

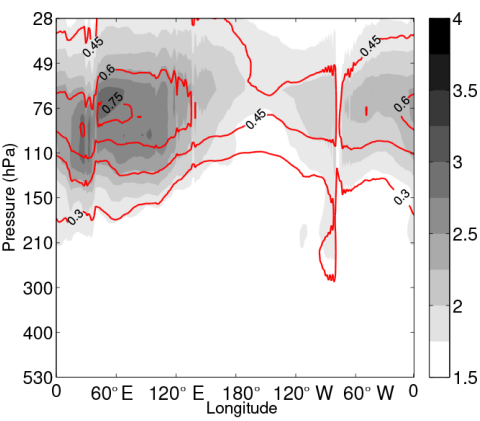

(c) 3-20 days

Figure 7. Longitude-pressure sections along $0.7^{\circ} \mathrm{N}$ of the $\mathrm{KW}$ zonal wind and temperature averaged over the 6-year period for (a) lowfrequency, (b) intra-seasonal and (c) intra-monthly periods. The contouring is as follows: (a) zonal wind is coloured each $1.5 \mathrm{~m} \mathrm{~s} \mathrm{~s}^{-1}$ and temperature is shown by red contours each $0.5 \mathrm{~K}$, with zero lines omitted; (b) absolute amplitudes of the zonal wind and temperature are shown in grey shades each $0.5 \mathrm{~m} \mathrm{~s}^{-1}$ and red contours, each $0.15 \mathrm{~K}$, respectively and (c) absolute amplitudes of the zonal wind are in grey shades each $0.25 \mathrm{~m} \mathrm{~s}^{-1}$ and temperature in red contours (each $0.15 \mathrm{~K}$ ).

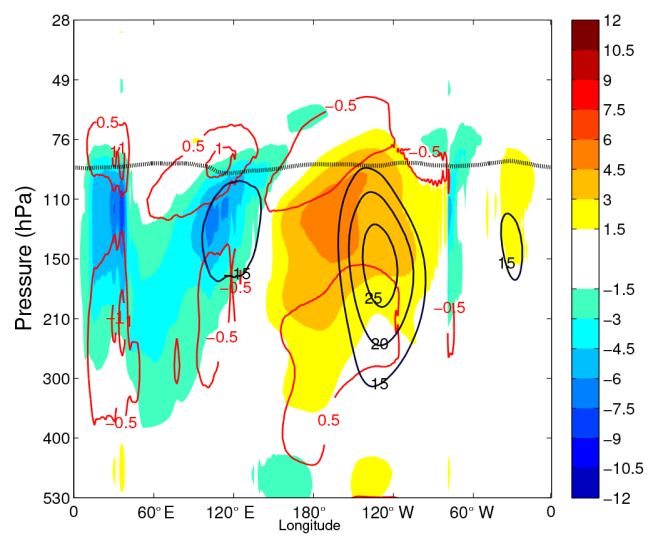

(a) DJF

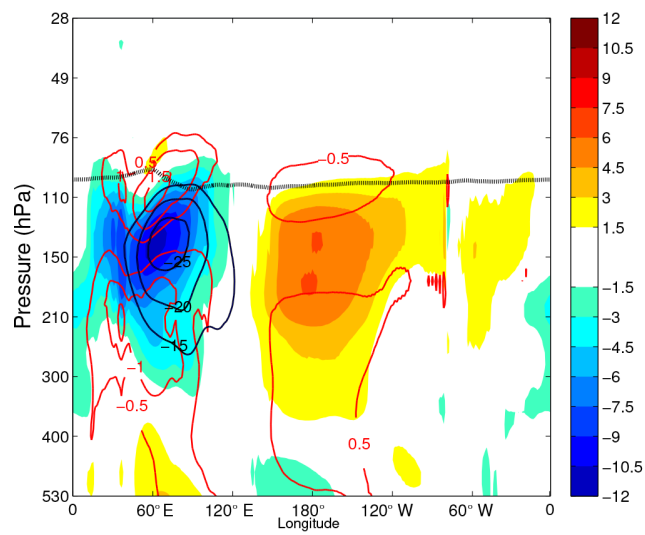

(c) JJA

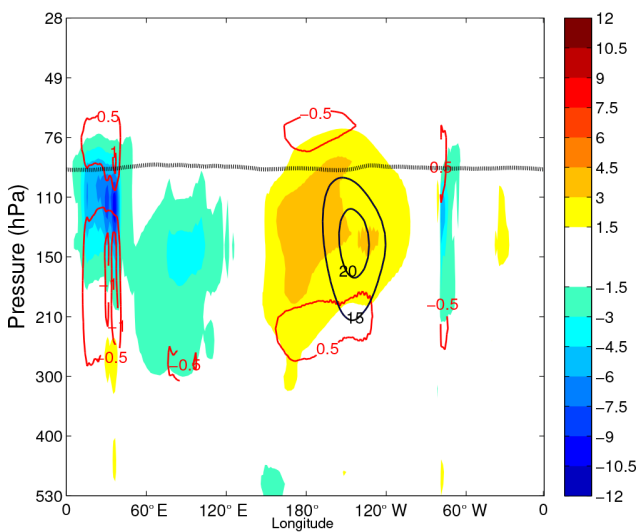

(b) MAM

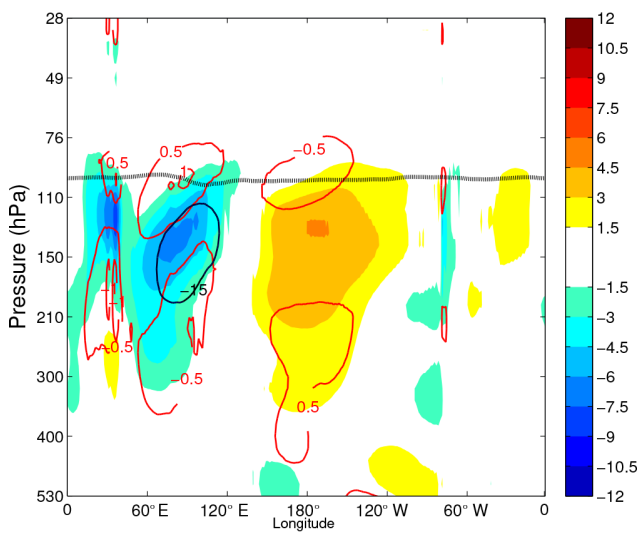

(d) SON

Figure 8. Seasonally averaged longitude-pressure sections of the KW zonal wind (blue-to-red colour-filled contours) and temperature (red contours) along $0.7^{\circ} \mathrm{N}$. (a) DJF, (b) MAM, (c) JJA and (d) SON. Contouring of the KW signal is the same as in Fig. 7a. A single static stability contour with value $5 \times 10^{-4} \mathrm{~s}^{-2}$ is shown as a thick dotted black line to represent the seasonal movement of the tropical tropopause height. The average background zonal wind is shown by blue contours (each $5 \mathrm{~m} \mathrm{~s}^{-1}$, starting from $15 \mathrm{~m} \mathrm{~s}^{-1}$ ). The background zonal wind and stability fields are latitudinally averaged over $5^{\circ} \mathrm{S}-5^{\circ} \mathrm{N}$. All fields are smoothed using a low-pass filter with the cut-off period of 90 days. 
found higher up at approximately $90 \mathrm{hPa}$. Values are somewhat weaker compared to Northern Hemisphere summer with easterlies up to $-6 \mathrm{~m} \mathrm{~s}^{-1}$ and westerlies up to $5 \mathrm{~m} \mathrm{~s}^{-1}$.

Finally, SON and MAM season months are transition seasons with respect to the strength and position of the KW dipole as it moves west- and downward towards JJA and eastand upward towards DJF. Season MAM has the weakest KW dipole with slightly stronger westerly winds up to $5 \mathrm{~m} \mathrm{~s}^{-1}$.

The longitudinal position and the strength of the lowfrequency KWs have been linked to the seasonal patterns of the background winds in the TTL representing the upper level monsoon and Walker circulations (Flannaghan and Fueglistaler, 2013). The average background winds maximize at $150 \mathrm{hPa}$ as shown in Fig. 3a. In Fig. 8, one can see how the KW easterlies in the eastern hemisphere are strongest during JJA in relation to the Indian and South Asian monsoon circulation. Background easterlies as strong as $-30 \mathrm{~m} \mathrm{~s}^{-1}$ are located approximately $10^{\circ}$ east of the $\mathrm{KW}$ maximum easterlies. Season DJF has the strongest background westerlies in relation to the upper-level circulation of the western Pacific anticyclones. Season MAM shows similar background wind patterns compared to DJF but with weaker circulation. Season SON shows similar patterns with JJA but with weaker winds.

Further details on longitudinal position and interannual variability in the low-frequency $\mathrm{KW}$ response at its maximum value at $150 \mathrm{hPa}$ are illustrated by the Hovmöller diagram in Fig. 9. For comparison, tropical convection is represented as well through the OLR proxy variable averaged over $15^{\circ} \mathrm{S}-15^{\circ} \mathrm{N}$ latitudes. All fields have been filtered with a 90-day cut-off low-pass filter in order to highlight the seasonality. As a result, one can observe enhanced or reduced $\mathrm{KW}$ activity during the same individual seasons as seen from the time series in Fig. 5. Above-average seasonal KW activity with stronger dipole structures occurred during the summer of 2007 (mainly through its easterlies at $60^{\circ} \mathrm{E}$ ) and during the winters of 2006-2007 and 2009-2010. In these winters, El Niño was active and a clear longitudinal eastward shift is observed in OLR and in the background circulation (not shown), as well as in the dipole KW structure. The El Niño winter of 2009-2010 was followed by a strong La Niña winter with an increase in tropical convection over the Maritime Continent (note OLR values below $195 \mathrm{Wm}^{-2}$ ).

The vertical seasonal movement of the $\mathrm{KW}$ dipole has been linked with the seasonal movement of the tropical tropopause height (Flannaghan and Fueglistaler, 2013; Ryu et al., 2008). The position of the tropical tropopause height (represented by a static stability value of $5 \times 10^{-4} \mathrm{~s}^{-2}$ in Fig. 8) is found at approximately $85 \mathrm{hPa}$ during DJF and descends towards $100 \mathrm{hPa}$ in JJA, similar to values obtained from GPS-RO observations by Grise et al. (2010). In particular, during JJA, one can notice how the asymmetry in the tropical tropopause height over the Indian Ocean around $60^{\circ} \mathrm{E}$ coincides with increasing temperatures by the

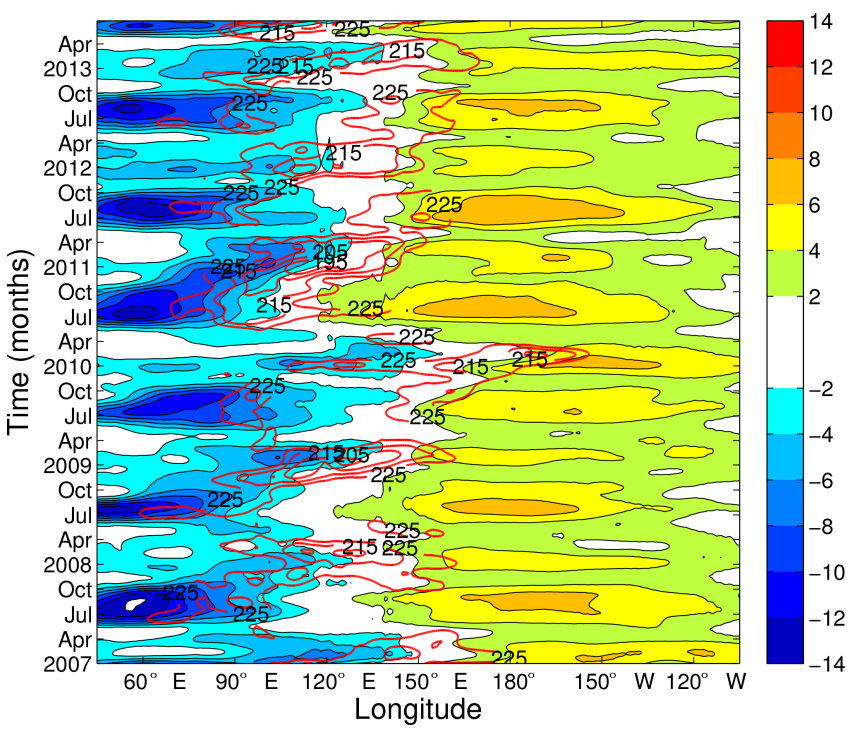

Figure 9. Longitude-time section at model level $45(\sim 153 \mathrm{hPa})$ of the $\mathrm{KW}$ zonal wind along $0.7^{\circ} \mathrm{N}$ (blue to red shaded contours every $2 \mathrm{~m} \mathrm{~s}^{-1}$ with zero line omitted) and the outgoing longwave radiation (OLR) averaged over the latitude belt $15^{\circ} \mathrm{S}-15^{\circ} \mathrm{N}$ (red contours each $10 \mathrm{Wm}^{-2}$ starting at $225 \mathrm{Wm}^{-2}$ ). Both fields have been filtered a priori using a low-pass filter with a cut-off period of 90 days.

$\mathrm{KW}$ dipole up to $1.5 \mathrm{~K}$. Such deformation of the tropical tropopause is also evident during DJF and SON seasons.

Figure 10a and b illustrate seasonal-mean KW temperatures ${\overline{T_{\mathrm{kw}, 1}}}^{S}$ in relation to the tropical tropopause layer defined by static stability $N^{2}$. Seasonal variations in KW temperatures are colocated with the position of the tropopause, descending down from its highest position during DJF to its lowest position during JJA. Temperature amplitudes are observed to decline roughly above $N^{2}=5-6 \times 10^{-4} \mathrm{~s}^{-2}$. Within this zonal-mean seasonal picture, zonal asymmetries in $N^{2}$ exist and are found: (i) near the International Date Line with values of $8 \times 10^{-4} \mathrm{~s}^{-2}$ at $80 \mathrm{hPa}$ during DJF and $7 \times 10^{-4} \mathrm{~s}^{-2}$ at $90 \mathrm{hPa}$ during JJA and (ii) lower at $100 \mathrm{hPa}$ over the Indian Ocean during JJA. Particularly during JJA, the deformation of the zonal-mean static stability field colocates strongly with the position of a strong $\mathrm{KW}$ temperature anomaly over the Indian Ocean. A rough estimation is made on the contribution of the KW anomaly to the zonal deformation of the tropopause layer by removing zonal-mean parts of both fields. First, static stability zonal anomalies, ${\overline{N^{\prime 2}}}^{s}$, are derived by subtracting zonal-mean values of $N^{2}$ from the full $N^{2}$ field per time step and at every pressure level, followed by seasonal averaging. Next, we can estimate the static stability change associated with the KW anomaly, using the relation $N_{\mathrm{kw}}^{2}=\frac{g}{\theta} \frac{\partial \theta_{\mathrm{kw}}}{\partial z}$, followed by seasonal averaging as well, i.e. ${\overline{N_{\mathrm{kw}}^{2}}}^{s}$. 


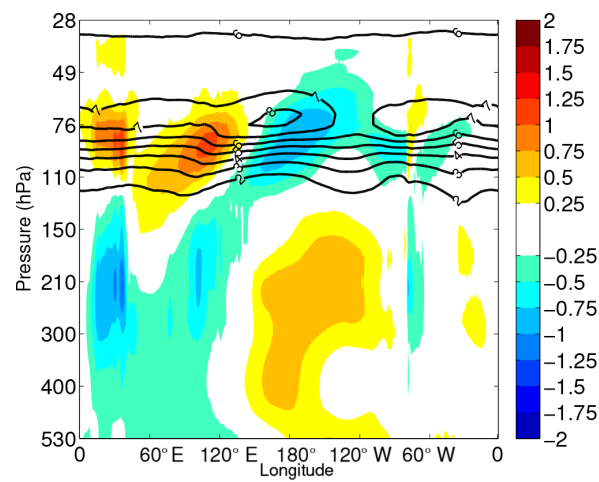

(a) DJF

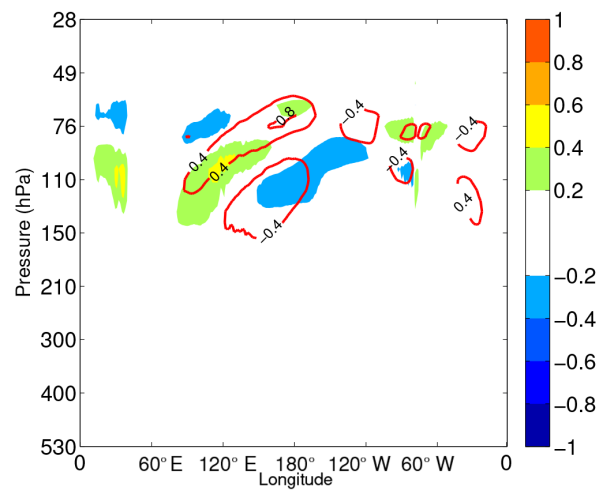

(c) DJF - $N^{2}$ anomalies

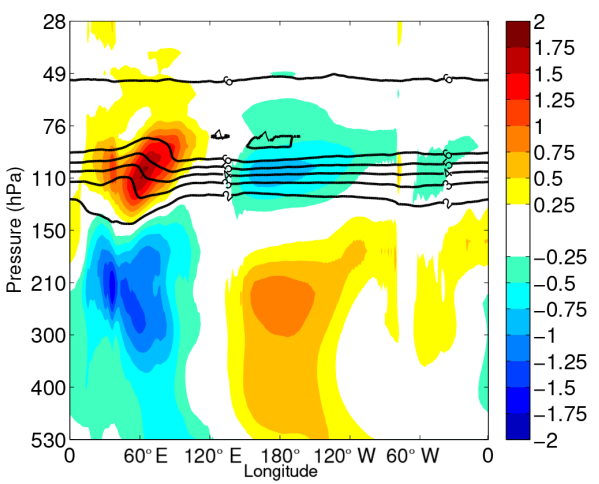

(b) JJA

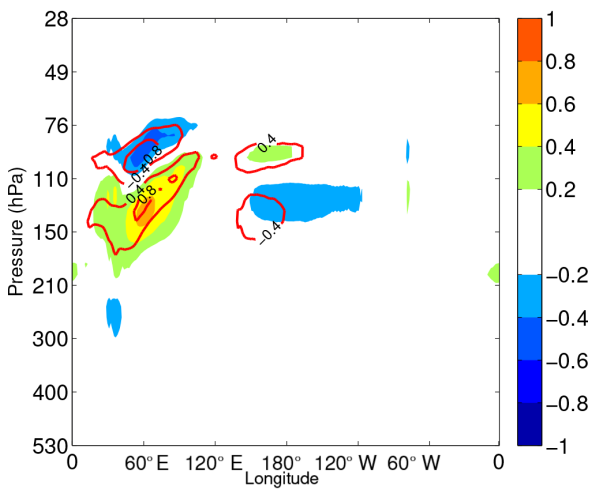

(d) JJA - $N^{2}$ anomalies

Figure 10. Seasonally averaged longitude-pressure sections for (a, c) DJF and (b, d) JJA. (a-b) KW temperature, $\overline{T_{\mathrm{kw}}} s$, (blue-to-red shades every $0.25 \mathrm{~K}$ ) and static stability field, $\overline{N^{2}}$ (black contours, each $1 \times 10^{-4} \mathrm{~s}^{-2}$, starting at $2 \times 10^{-4} \mathrm{~s}^{-2}$ ). (c-d) KW static stability anomaly, ${\overline{N_{\mathrm{kw}}^{2}}}^{s}$ (blue-to-red, each $0.2 \times 10^{-4} \mathrm{~s}^{-2}$ ), and static stability anomaly with respect to the zonal mean, $\overline{N^{\prime 2}} s$ (red contours, each $0.4 \times$ $\left.10^{-4} \mathrm{~s}^{-2}\right)$.

As a result, Fig. 10c and d show how both static stability anomalies are overlapping. During DJF, the structure of the zonal anomaly ${\overline{N^{\prime 2}}}^{S}$ has a positively valued tilt eastward which stretches up to $80 \mathrm{hPa}$, while during JJA a strong static stability anomaly is found more localized over the Indian Ocean region with values in the TTL up to ${\overline{N^{\prime 2}}}^{\mathrm{JJA}}=$ $\pm 0.8 \times 10^{-4} \mathrm{~s}^{-2}$. The anomaly associated with the KW temperature is found to peak up to $+0.6 \times 10^{-4} \mathrm{~s}^{-2}$ during JJA and up to $+0.4 \times 10^{-4} \mathrm{~s}^{-2}$ during DJF. Finally, by dividing both fields with each other, the resulting contribution of the quasi-stationary $\mathrm{KW}$ to the observed deformation of the tropical tropopause layer is estimated up to $60 \%$ during JJA and $80 \%$ during DJF.

\subsection{Intra-seasonal $\mathrm{KW}$ variability}

The seasonality of intra-seasonal KW variability is shown in Fig. 11 and shall be briefly discussed here. The DJF stands out as the most active season for KW activity, located mainly in the eastern hemisphere centred at $100^{\circ} \mathrm{E}$ and with maximum activity at $110 \mathrm{hPa}$ for zonal wind and temperature with a second maximum in temperature at $90 \mathrm{hPa}$. Values observed are up to $0.8 \mathrm{~K}$ for $\mathrm{KW}$ temperature and $5 \mathrm{~m} \mathrm{~s}^{-1}$ for KW zonal wind. During the MAM season, the KW activity fields are weaker but spread over a larger area in the eastern hemisphere and in the TTL with maximum activity centred at $120 \mathrm{hPa}(90 \mathrm{hPa})$ for the zonal wind (temperature) component. Both JJA and SON seasons have KW activity positioned at lower altitudes and more westward. In both seasons, KW zonal wind activity is split up between two structures with an eastward tilt with height: one with a maximum around $110^{\circ} \mathrm{E}$ and one pattern starting from $100 \mathrm{hPa}$ and extending towards $60^{\circ} \mathrm{E}$. Note also the increase in $\mathrm{KW}$ activity in the western hemisphere below $150 \mathrm{hPa}$ in the east Pacific. The maximum KW activity in the temperature component for both seasons is positioned near $100 \mathrm{hPa}$ approximately on the tropical tropopause contour with a value $5 \times 10^{-4} \mathrm{~s}^{-2}$.

The eastward tilted structure is observed throughout all seasons except MAM when background easterly winds are nearly absent in the eastern hemisphere. In all other seasons one can observe how the tilted structure is locked to the background easterlies with maximum amplitudes located slightly above and west of it. Such eastward tilt with height has been 


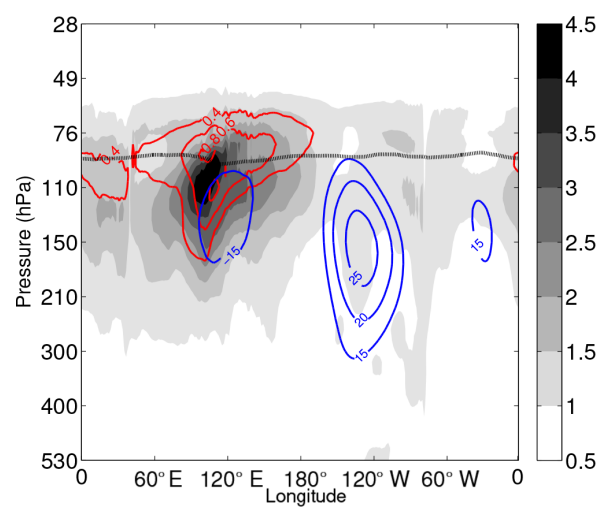

(a) DJF

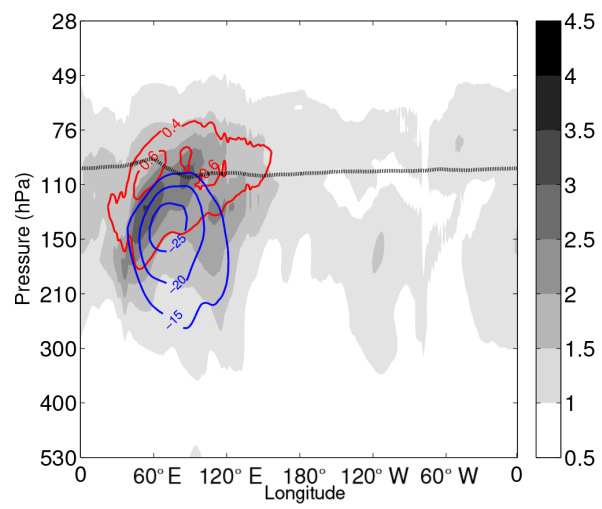

(c) JJA

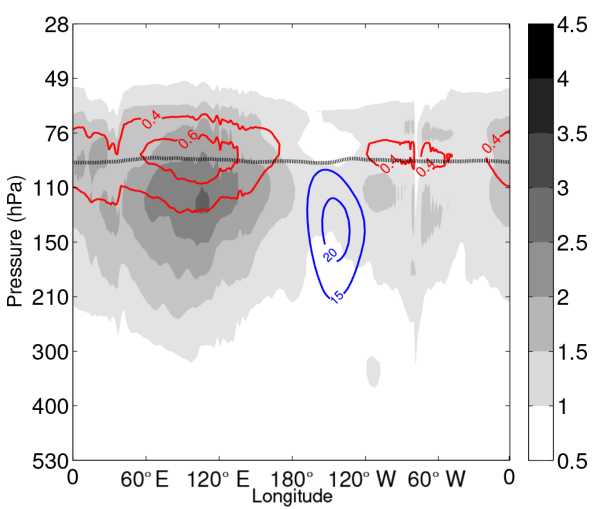

(b) MAM

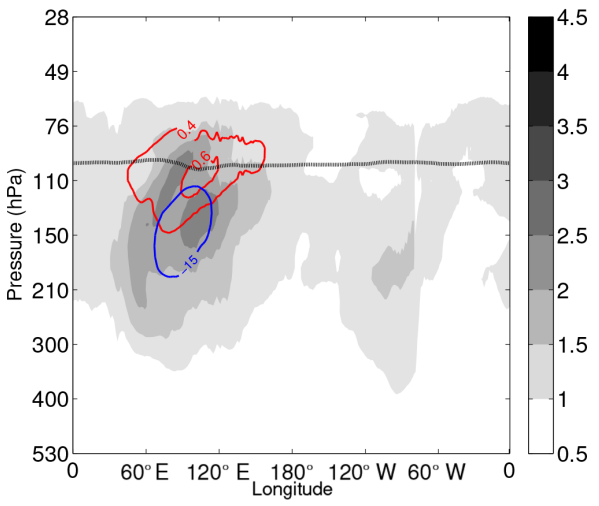

(d) SON

Figure 11. Seasonally averaged longitude-pressure sections along $0.7^{\circ} \mathrm{N}$ of the intra-seasonal KW zonal wind (white-to-black shades, each $0.5 \mathrm{~m} \mathrm{~s}^{-1}$ ) and temperature (red contours, each 0.2 K). (a) DJF, (b) MAM, (c) JJA and (d) SON. The averaging is performed for the absolute values of both zonal wind and temperature perturbations. The background zonal wind (shown by blue contours) and the tropical tropopause height (single thick dotted contour) are defined as in Fig. 8.

frequently observed, for example over a radiosonde station Medan at $100^{\circ} \mathrm{E}$ during the early stage of Madden-Julian Oscillation development (Kiladis et al., 2005).

\subsection{Intra-monthly KWs}

The seasonal variability in intra-monthly KWs, represented by their absolute amplitudes $\overline{\left|u_{\mathrm{kw}, \mathrm{h}}^{\prime}\right|}$ s and $\overline{\left|T_{\mathrm{kw}, \mathrm{h}}^{\prime}\right|}$, shall be examined in relation to the background conditions. Figure 12 illustrates favourable regions for $\mathrm{KW}$ activity. In general, $\mathrm{KW}$ activity increases upward from around $120 \mathrm{hPa}$ towards its zonal-mean peak value at $76 \mathrm{hPa}$. The largest values are observed in the eastern hemisphere in a region from 30 to $150^{\circ} \mathrm{E}$. The temperature component in particular has a constant maximum peak (up to $0.8 \mathrm{~K}$ ) located around $76 \mathrm{hPa}$ throughout the year, where the largest increase in $N^{2}$ also occurs as shown in Fig. 3. Above $70 \mathrm{hPa}, \mathrm{KW}$ activity continuously decreases in the stratosphere.

The longitudinal structure of the KW zonal wind shows two distinct peaks in the TTL, one consistently located at $76 \mathrm{hPa}$ and another around $100-110 \mathrm{hPa}$ in the eastern hemisphere, which is mainly present during solstice seasons. The first maximum coincides with the temperature distribution, which can be explained by their balance relationships and free horizontal propagation in the stratosphere. Below the tropopause, KW activity is coupled to convective processes alternating the tropospheric vertical wave structures as discussed by Flannaghan and Fueglistaler (2012).

The secondary maximum around $110 \mathrm{hPa}$ in Fig. 12 is present mainly during solstice seasons in the eastern hemisphere and it is associated with the seasonal movement of the background wind.

The maximum of $\mathrm{KW}$ wind and the background wind maximum move eastward from DJF to JJA seasons similar to the low-frequency variability.

A day-by-day comparison of the KW activity and background wind confirms that propagating KWs amplify while approaching a region of strong easterlies, forming a folding structure around it while the individual KWs dissipate towards the centre of easterly winds. In Fig. 12 one can notice a fast reduction of KW amplitudes eastward of its maximum towards the centre of the background easterlies. It is likely related to dissipation and wave breaking processes as observed over Indonesia (120 E) by Fujiwara et al. (2003). 


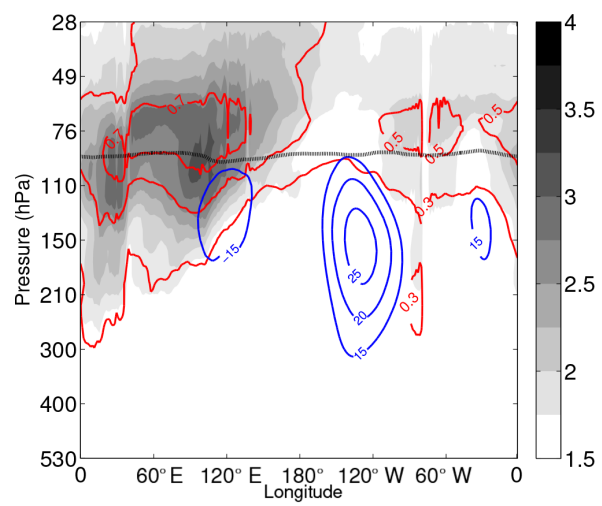

(a) DJF

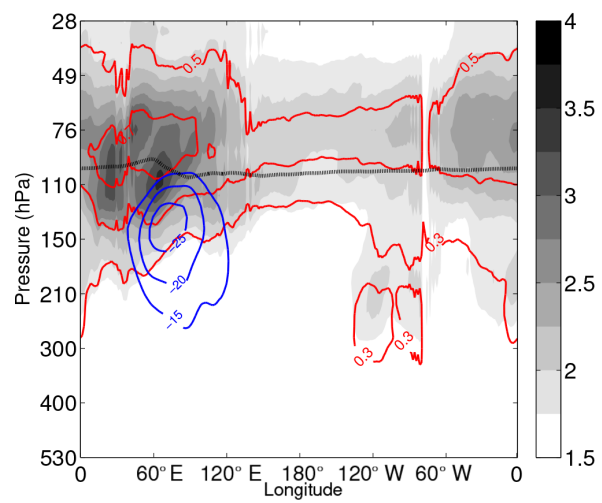

(c) JJA

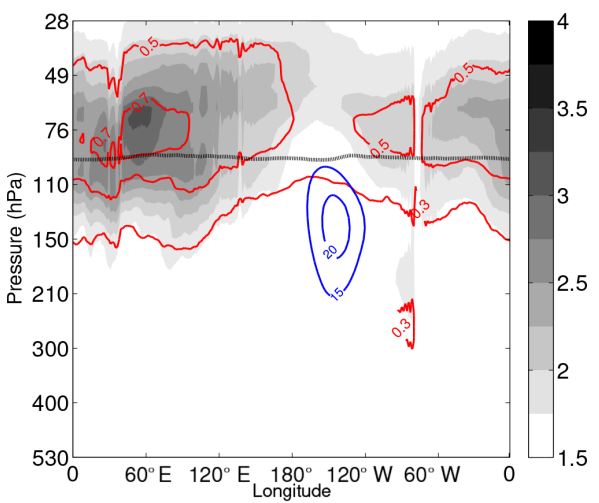

(b) MAM

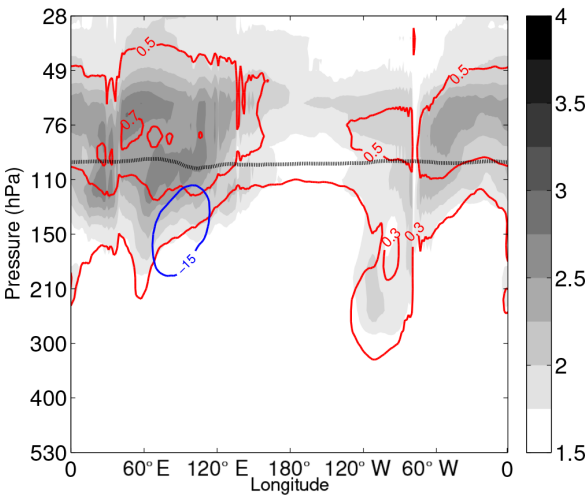

(d) SON

Figure 12. As in Fig. 11 but for the intra-monthly KWs. The zonal wind (white-to-black shades) is drawn every $0.25 \mathrm{~m} \mathrm{~s}^{-1}$.

Within such regions, the KW-background-wind interaction becomes complex and the linearity assumption breaks (Ryu et al., 2008; Flannaghan and Fueglistaler, 2013).

A comparison with the previous study by Suzuki and Shiotani (2008) using ERA-40 data shows that the L91 data contain stronger KW activity in the vicinity of the background easterlies in the eastern hemisphere, and more finescale details, which can be explained by better analyses based on more observations and improved models including increased resolution. For example, Suzuki and Shiotani (2008) used 5 levels of ERA-40 data between 50 and $200 \mathrm{hPa}$ whereas the present study considers 25 model levels between 50-200 hPa. Maxima of the KW temperature signal appear in similar locations and strength, except for a small offset in vertical position $(70 \mathrm{hPa}$ in Suzuki and Shiotani, 2008, versus $80 \mathrm{hPa}$ in Fig. 12) and a larger zonal asymmetry in our results.

Another view of the seasonal cycle of free-propagating KWs is illustrated in Fig. 13, which focuses on the spatiotemporal distribution of individual KW tracks. Hovmöller diagrams are illustrated for $\mathrm{KW}$ zonal wind and temperature at levels 110 and $200 \mathrm{hPa}$ cumulated from different years into a single calendar year along with the background zonal wind. In addition, the monthly-mean values of daily maximum $\mathrm{KW}$ amplitudes occurring in longitude are added on the right side of each diagram. It represents seasonality in the KW maximum amplitudes in a similar fashion to Fig. 6 in Alexander and Ortland (2010), which is based on HIRDLS satellite data.

The individual wave tracks at $110 \mathrm{hPa}$ illustrate KWs with amplitudes exceeding $3 \mathrm{~m} \mathrm{~s}^{-1}$ and $0.6 \mathrm{~K}$, which are propagating throughout the year in the eastern hemisphere, during June-October months only over the Pacific, and all except DJF months in most of the western hemisphere. Typical wave tracks start east of the $0^{\circ}\left(30^{\circ} \mathrm{W}\right)$ meridian during winter (summer) and largely disappear west of $120^{\circ} \mathrm{E}$. The largest wave amplitudes are observed between 50 and $100^{\circ} \mathrm{E}$ prior to regions of easterly winds in agreement with Fig. 12. Here, presented details show that the most notable waves appear during the Asian monsoon period with upper-level easterlies prevailing from June into September. The largest KW amplitudes appear to be confined to the June and July months followed by a rapid drop in August. In fact, a local minimum in the number of KWs as well as in wave amplitudes occurs in August before the KW activity increases slightly during autumn.

At $200 \mathrm{hPa}$, the favourable area for KW propagation shifts to the western hemisphere and high KW activity is observed west of the South American continent throughout the year 


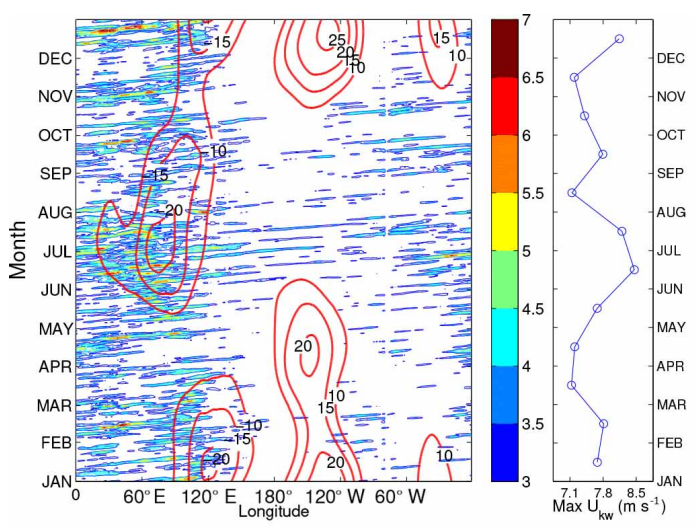

(a) $\left|u_{k w}^{\prime}\right|-110 \mathrm{hPa}$

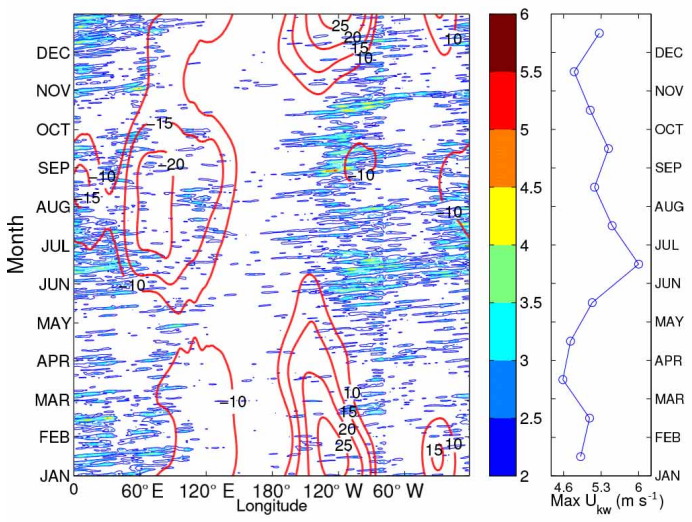

(c) $\left|u_{k w}^{\prime}\right|-200 \mathrm{hPa}$

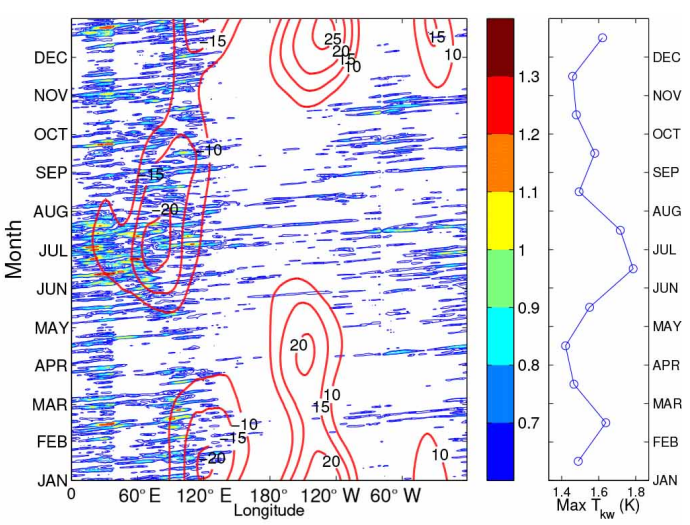

(b) $\left|T_{k w}^{\prime}\right|-110 \mathrm{hPa}$

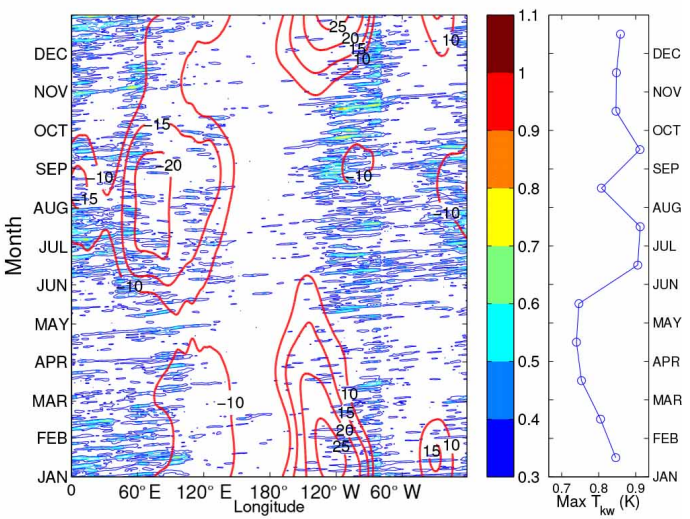

(d) $\left|T_{k w}^{\prime}\right|-200 \mathrm{hPa}$

Figure 13. Intra-monthly KW zonal wind and temperature composites as a function of longitude and month in a calendar year at (ab) model level $40(\sim 110 \mathrm{hPa})$ and $(\mathbf{c}-\mathbf{d})$ model level $49(\sim 200 \mathrm{hPa})$ along $0.7^{\circ} \mathrm{N}$. The waves are accumulated from different years into a single calendar year to highlight seasonal behaviour. Only the most energetic signals are shown: (a, c) zonal wind, $\left|u_{\mathrm{kw}}^{\prime}\right|$, each $0.5 \mathrm{~m} \mathrm{~s}{ }^{-1}$, and (b, d) temperature $\left|T_{\mathrm{kw}}^{\prime}\right|$, each $0.1 \mathrm{~K}$. For comparison, the background zonal wind field is presented by red contours, each $5 \mathrm{~m} \mathrm{~s}{ }^{-1}$. On the right side of each panel, blue lines with circles denote maximal amplitude of the KW zonal wind occurring anywhere along the equator averaged over the 6-year period for each calendar month. This highlights seasonality in the maximum amplification of propagating KWs.

(west of $80^{\circ} \mathrm{W}$ ) with a westward extension over the Pacific during JJA. Another set of wave tracks starts over equatorial South America around $30^{\circ} \mathrm{W}$ and continues until $60^{\circ} \mathrm{E}$ during JJA. During DJF these wave tracks shift more east and start at $5^{\circ} \mathrm{W}$ and continue until $90^{\circ} \mathrm{E}$. The seasonal shifts of approximately $30^{\circ}$ in $\mathrm{KW}$ tracks colocate with similar shifts in the prevailing TTL winds.

The amplitude of KWs undergoes a clear annual cycle with a small secondary peak present during DJF, as represented by the monthly-means of daily maximum amplitudes on the right side of Fig. 13. The largest amplitudes are found at $110 \mathrm{hPa}$ during JJA with monthly-mean zonal wind (temperature) values up to $8.5 \mathrm{~m} \mathrm{~s}^{-1}(1.8 \mathrm{~K})$ in June. During the DJF months KWs amplify more eastward with monthly-mean zonal wind (temperature) values up to $7.8 \mathrm{~m} \mathrm{~s}^{-1}(1.6 \mathrm{~K})$ in December. Our result matches well with the observed seasonal pattern in maximum $\mathrm{KW}$ temperatures at $16 \mathrm{~km}(\sim 100 \mathrm{hPa})$ from the HIRDLS satellite observations
(Alexander and Ortland, 2010, Fig. 6). At $200 \mathrm{hPa}$, KW amplitudes are on average lower with a yearly-averaged amplitude reduction around $55 \%$ in temperature and $35 \%$ in zonal wind.

The semiannual cycle in maximum amplitudes remains visible up until $70 \mathrm{hPa}$. Above $70 \mathrm{hPa}$, where the $\mathrm{KW}$ activity remains large in the eastern hemisphere (Fig. 12), the semiannual cycle is replaced by an interannual cycle in line with the dominant impact of the QBO.

\section{Discussion and conclusions}

We have applied a multivariate decomposition of the ECMWF operational analyses during the period 2007-2013 when the operational data assimilation and forecasting were performed on 91 model levels. The applied normal-mode function decomposition simultaneously provides the wind components, geopotential height and temperature perturba- 
tions of Kelvin waves (KWs) on many scales without any prior data filtering. The three-dimensional $\mathrm{KW}$ structure in the upper troposphere and lower stratosphere is composed of KW solutions of 60 linearized shallow-water equation systems on the sphere with equivalent depths from $10 \mathrm{~km}$ up to about $3 \mathrm{~m}$. As the $\mathrm{KW}$ meridional wind component is very small it is not discussed here. We showed that large-scale KWs readily persist in the data despite analysing selected processing times independently.

The KW is a normal mode of the global atmosphere and our 3-D-orthogonal decomposition allows for the quantification of its contribution to the global energy spectrum and variability. We have presented the total (kinetic + potential) energy of KWs in the L91 data as a function of the zonal wavenumber in different seasons. The zonal wavenumber $k=1$ contains the largest portion of KW energy in all seasons. There is almost one-third more energy in JJA than in MAM in $k=1$. In $k=2$ there is $50 \%$ less energy than in $k=1$ but season JJA still contains most energy. In all larger zonal wavenumbers, the most energetic season is DJF.

We focused on the spatiotemporal features of the KW temperature and zonal wind components in the four seasons. The KW seasonal cycle in the tropical tropopause layer (TTL) was compared with seasonal variability in the outgoing longwave radiation (OLR), and the background wind and stability fields, which are believed to play an important role in KW variability. Our results of the seasonal KW variability complement previous studies which applied different methods for the KW filtering and different datasets. The frequency spectrum has revealed a semiannual cycle as well as inter-seasonal and intra-monthly variability. Three ranges of wave periods were analysed: 3-20, 20-90 and longer than 90 days. This choice was partly deliberate in order to compare our results with several previous studies of KW variability. First we demonstrated that the low-frequency KW dipole pattern in the TTL, with westerly winds in the western hemisphere and with easterly winds in the eastern hemisphere, partly resembles a seasonal-averaged Gill-type "wave-1" pattern and contains partly low-frequency modulation of vertically propagating KWs. The quadrupole-shaped temperature component represents a thermally adjusted pattern with respect to the zonal wind component, and contributes to seasonal warming above $100 \mathrm{hPa}$ in the western and cooling in the eastern hemisphere. The largest KW amplitudes are observed during JJA and DJF seasons. From boreal summer towards winter, $\mathrm{KW}$ perturbations move eastward (from the Indian Ocean basin towards the Maritime Continent) and upward (e.g. zonal wind component moves up from 150 towards $120 \mathrm{hPa}$ ). The KW zonal wind amplitude varies between $12 \mathrm{~m} \mathrm{~s}^{-1}$ strong easterlies over the Indian Ocean near $150 \mathrm{hPa}$ in JJA and $6 \mathrm{~m} \mathrm{~s}^{-1}$ over the western Pacific. Over the Indian Ocean in JJA, the KW easterlies thus make up almost half of the total wind vector. The associated KW temperature perturbations are from $1.5 \mathrm{~K}$ over the Indian Ocean in JJA to $-0.5 \mathrm{~K}$ over the western Pacific. The zonal modula- tion of KWs is found to be locked with respect to the seasonal movement of convection and the convective outflow in the TTL. The modulation effect is strongest for the lowfrequency KWs during the summer monsoon season, when strong easterly winds are present at $150 \mathrm{hPa}$, resulting in the largest KW zonal wind and temperature anomalies, of which the latter results in deformation of the tropical tropopause over the Indian Ocean.

Intra-seasonal (periods 20-90 days) activity is strongest in DJF with maxima up to $0.8 \mathrm{~K}$ for $\mathrm{KW}$ temperature and up to $5 \mathrm{~m} \mathrm{~s}^{-1}$ for $\mathrm{KW}$ zonal wind centred at $120^{\circ} \mathrm{E}$. Both temperature and zonal wind activities have an eastward tilt with height. In comparison to a previous study by Suzuki and Shiotani (2008) using ERA-40 data, the slanted structure in the present data continues to extend more upward and eastward, which is likely due to the increased number of vertical model levels compared to ERA-40. The importance of vertical model resolution for the $\mathrm{KW}$ structure and amplitude was demonstrated in Žagar et al. (2012) and Podglajen et al. (2014).

For periods 3-20 days, the seasonal cycle of KWs is clearly seen in the wave amplitude. In the zonal-mean perspective, the largest amplitudes are located between 70 and $100 \mathrm{hPa}$ for both zonal wind and temperature but it is modulated by the seasonal movement of the TTL. A major zonal asymmetry was found in KW activity: around $110 \mathrm{hPa}$ the $\mathrm{KW}$ undergoes amplification mainly in the eastern hemisphere during the solstice seasons, while at $200 \mathrm{hPa}$ a secondary region of the $\mathrm{KW}$ amplification occurs in the western hemisphere during boreal summer. The inter-monthly KWs show largest amplitudes in the vicinity of the strongest easterlies preferably west and above the centre of the easterlies. The applied novel methodology makes it possible to observe such dynamics on a daily basis whenever easterlies are strong in the TTL. A nearly real-time representation of the KW activity is available at http://modes.fmf.uni-lj.si (last access: 12 June 2018).

In summary, our seasonal variability analysis shows that the background wind in the TTL linked with convective outflows play a dominant role in the longitudinal position where the zonal modulation of KWs is preferred, while the tropical tropopause and its seasonal vertical movement determine the vertical extent of the KW modulation processes.

Data availability. The operational ECMWF analyses on model levels can be obtained from the ECMWF archive. The applied MODES software is accessible through http://modes.fmf.uni-lj.si (last access: 12 June 2018). The output Kelvin wave geopotential and winds in L91 ECMWF analyses are available from the authors upon request.

Competing interests. The authors declare that they have no conflict of interest. 
Acknowledgements. This study was funded by the European Research Council (ERC), grant agreement no. 280153, MODES. We are grateful to George Kiladis and an anonymous reviewer for their detailed and constructive comments.

Edited by: Timothy J. Dunkerton

Reviewed by: George Kiladis and one anonymous referee

\section{References}

Alexander, M. J. and Ortland, D. A.: Equatorial waves in High Resolution Dynamics Limb Sounder (HIRDLS) data, J. Geophys. Res., 115, D24111, https://doi.org/10.1029/2010JD014782, 2010.

Andrews, D. G., Holton, J. R., and Leovy, C. B.: Middle atmospheric dynamics, Academic Press, 1987.

Baldwin, M. P., Gray, L. J., Dunkerton, T. J., Hamilton, K., Haynes, P. H., Randel, W. J., Holton, J. R., Alexander, M. J., Hirota, I., Horinouchi, T., Jones, D. B. A., Kinnersley, J. S., Marquardt, C., Sato, K., and Takahashi, M.: The Quasi-Biennial Oscillation, Rev. Geophys., 39, 179-229, 2001.

Boyd, J. P.: The Effects of Latitudinal Shear on Equatorial Waves. Part II: Applications to the Atmosphere, J. Atmos. Sci., 35, 2259-2267, 1978.

Boyd, J. P.: Dynamics of the Equatorial Ocean, Springer-Verlag GmbH Germany, 2018.

Boyd, J. P. and Zhou, C.: Uniform Asymptotics for the Linear Kelvin Wave in Spherical Geometry, J. Atmos. Sci., 65, 655-660, https://doi.org/10.1175/2007JAS2356.1, 2008.

Ern, M. and Preusse, P.: Wave fluxes of equatorial Kelvin waves and QBO zonal wind forcing derived from SABER and ECMWF temperature space-time spectra, Atmos. Chem. Phys., 9, 39573986, https://doi.org/10.5194/acp-9-3957-2009, 2009.

Ern, M., Preusse, P., Krebsbach, M., Mlynczak, M. G., and Russell III, J. M.: Equatorial wave analysis from SABER and ECMWF temperatures, Atmos. Chem. Phys., 8, 845-869, https://doi.org/10.5194/acp-8-845-2008, 2008.

Flannaghan, T. J. and Fueglistaler, S.: Tracking Kelvin waves from the equatorial troposphere into the stratosphere, J. Geophys. Res., 117, D21108, https://doi.org/10.1029/2012JD017448, 2012.

Flannaghan, T. J. and Fueglistaler, S.: The importance of the tropical tropopause layer for equatorial Kelvin wave propagation, J. Geophys. Res., 118, 5160-5175, 2013.

Fueglistaler, S., Dessler, A. E., Dunkerton, T. J., Folkins, I., Fu, Q., and Mote, P. W.: Tropical tropopause layer, Rev. Geophys., 47, RG1004, https://doi.org/10.1029/2008RG000267, 2009.

Fujiwara, M., Yamamoto, M. K., Hashiguchi, H., and Horinouchi, T.: Turbulence at the tropopause due to breaking Kelvin waves observed by the Equatorial Atmosphere Radar, Geophys. Res. Lett., 30, 1171, https://doi.org/10.1029/2002GL016278, 2003.

Garcia, R. R. and Salby, M. L.: Transient response to localized episodic heating in the Tropics. Part II: Far-field behavior, J. Atmos. Sci., 44, 499-530, 1987.

Garcia, R. R., Lieberman, R., Russell III, J. M., and Mlynczak, M. G.: Large-scale waves in the mesosphere and lower thermosphere observed by SABER, J. Atmos. Sci., 62, 4384-4399, https://doi.org/10.1175/JAS3612.1, 2005.
Gill, A. E.: Some simple solution for heat-induced tropical circulation, Q. J. Roy. Meteor. Soc., 106, 447-462, 1980.

Gill, A. E.: Atmosphere-Ocean Dynamics, Academic Press, New York, 1982.

Grise, K. M., Thompson, D. W. J., and Birner, T.: A global survey of static stability in the stratosphere and upper troposphere, J. Climate, 23, 2275-2292, 2010.

Hayashi, Y.: Space-time spectral analysis and its applications to atmospheric waves, J. Meteorol. Soc. Jpn., 60, 156-171, 1982.

Highwood, E. J. and Hoskins, B. J.: The tropical tropopause, Q. J. Roy. Meteor. Soc., 124, 1579-1604, 1998.

Holton, J. R. and Lindzen, R. S.: An updated theory for the quasibiennial cycle of the tropical stratosphere, J. Atmos. Sci., 29, 1076-1080, 1972.

Kasahara, A.: Normal modes of ultralong waves in the atmosphere, Mon. Weather Rev., 104, 669-690, 1976.

Kasahara, A.: Effect of zonal flows on the free oscillations of a barotropic atmosphere, J. Atmos. Sci., 37, 917-929, Corrigendum: 38, 2284-2285, 1980.

Kasahara, A. and Puri, K.: Spectral representation of threedimensional global data by expansion in normal mode functions, Mon. Weather Rev., 109, 37-51, 1981.

Kedzierski, R. P., Matthes, K., and Bumke, K.: The tropical tropopause inversion layer: variability and modulation by equatorial waves, Atmos. Chem. Phys., 16, 11617-11633, https://doi.org/10.5194/acp-16-11617-2016, 2016.

Kiladis, G. N., Straub, K. H., and Haertel, P. T.: Zonal and vertical structure of the Madden-Julian Oscillation, J. Atmos. Sci., 62, 2790-2809, https://doi.org/10.1175/JAS3520.1, 2005.

Liebmann, B. and Smith, C. A.: Description of a complete (interpolated) outgoing longwave radiation dataset, B. Am. Meteorol. Soc., 77, 1275-1277, 1996.

Lin, J.-L., Kiladis, G. N., Mapes, B. E., Weickmann, K. M., Sperber, K. R., Lin, W., Wheeler, M. C., Schubert, S. D., Genio, A. D., Donner, L. J., Emori, S., Gueremy, J.-F., Hourdin, F., Rasch, P. J., Roeckner, E., and Scinocca, J. F.: Tropical intraseasonal variability in 14 IPCC AR4 climate models. Part I: Convective signals, J. Climate, 19, 2665-2690, 2006.

Matsuno, T.: Quasi-geostrophic motions in the equatorial area, J. Meteorol. Soc. Jpn., 44, 25-43, 1966.

Podglajen, A., Hertzog, A., Plougonven, R., and Žagar, N.: Assessment of the accuracy of (re)analyses in the equatorial lower stratosphere, J. Geophys. Res.-Atmos., 119, 11166-11188, https://doi.org/10.1002/2014JD021849, 2014.

Randel, W. J. and Wu, F.: Kelvin wave variability near the equatorial tropopause observed in GPS radio occultation measurements, J. Geophys. Res., 105, 15509-15523, https://doi.org/10.1029/2000JD900155, 2005.

Ryu, J.-H., Lee, S., and Son, S.-W.: Vertically propagating Kelvin Waves and tropical tropopause variability, J. Atmos. Sci., 65, 1817-1837, 2008.

Salby, M. L. and Garcia, R. R.: Transient response to localized episodic heating in the tropics. Part I: Excitation and short-time near-field behavior, J. Atmos. Sci., 44, 458-498, 1987.

Shumway, R. and Stoffer, D.: Time series analysis and its applications: with $\mathrm{R}$ examples, Springer texts in statistics, Springer, New York, available at: https://books.google.si/books? id=dbS5IQ8P5gYC (last access: 1 March 2013), 2010. 
Son, S.-W., Lim, Y., Yoo, C., Hendon, H. H., and Kim, J.: Stratospheric Control of the Madden-Julian Oscillation, J. Climate, 30, 1909-1922, https://doi.org/10.1175/JCLI-D-16-0620.1, 2017.

Suzuki, J. and Shiotani, M.: Space-time variability of equatorial Kelvin waves and intraseasonal oscillations around the tropical tropopause, J. Geophys. Res., 113, D16110, https://doi.org/10.1029/2007JD009456, 2008.

Tindall, J. C., Thuburn, J., and Highwood, E. J.: Equatorial waves in the lower stratosphere. II: Annual and interannual variability, Q. J. Roy. Meteor. Soc., 132, 195-212, https://doi.org/10.1256/qj.04.153, 2006.

Tsai, H.-F., Tsuda, T., Hajj, G., Wickert, J., and Aoyama, Y.: Equatorial Kelvin waves observed with GPS occultation measurements (CHAMP and SAC-C), J. Meteorol. Soc. Jpn., 82, 397406, 2004.

Venkat Ratnam, M., Tsuda, T., Kozu, T., and Mori, S.: Longterm behavior of the Kelvin waves revealed by CHAMP/GPS RO measurements and their effects on the tropopause structure, Ann. Geophys., 24, 1355-1366, https://doi.org/10.5194/angeo24-1355-2006, 2006.

Žagar, N., Andersson, E., and Fisher, M.: Balanced tropical data assimilation based on a study of equatorial waves in ECMWF short-range forecast errors, Q. J. Roy. Meteor. Soc., 131, 9871011, https://doi.org/10.1256/qj.04.54, 2005.

Žagar, N., Andersson, E., Fisher, M., and Untch, A.: Influence of the quasi-biennial oscillation on the ECMWF model short-range forecast errors in the tropical stratosphere, Q. J. Roy. Meteor. Soc., 133, 1843-1853, 2007.
Žagar, N., Tribbia, J., Anderson, J. L., and Raeder, K.: Uncertainties of estimates of inertia-gravity energy in the atmosphere. Part II: Large-scale equatorial waves, Mon. Weather Rev., 137, 38583873, Corrigendum: 138, 2476-2477, 2009.

Žagar, N., Terasaki, K., and Tanaka, H. L.: Impact of the vertical resolution of analysis data on the estimates of large-scale inertiogravity energy, Mon. Weather Rev., 140, 2297-2307, 2012.

Žagar, N., Kasahara, A., Terasaki, K., Tribbia, J., and Tanaka, H.: Normal-mode function representation of global 3-D data sets: open-access software for the atmospheric research community, Geosci. Model Dev., 8, 1169-1195, https://doi.org/10.5194/gmd8-1169-2015, 2015.

Wallace, J. M. and Kousky, V. E.: Observational evidence of Kelvin waves in the tropical stratosphere, J. Atmos. Sci., 25, 900-907, 1968.

Wheeler, M. and Kiladis, G. N.: Convectively coupled equatorial waves: Analysis of clouds and temperature in the wavenumberfrequency domain, J. Atmos. Sci., 56, 374-399, 1999.

Yang, G.-Y. and Hoskins, B. J.: ENSO impact on Kelvin Waves and associated tropical convection, J. Atmos. Sci., 70, 3513-3532, 2013.

Yang, G.-Y., Hoskins, B. J., and Slingo, J.: Convectively coupled equatorial waves: A new methodology for identifying wave structures in observational data, J. Atmos. Sci., 60, 1637-1654, 2003. 